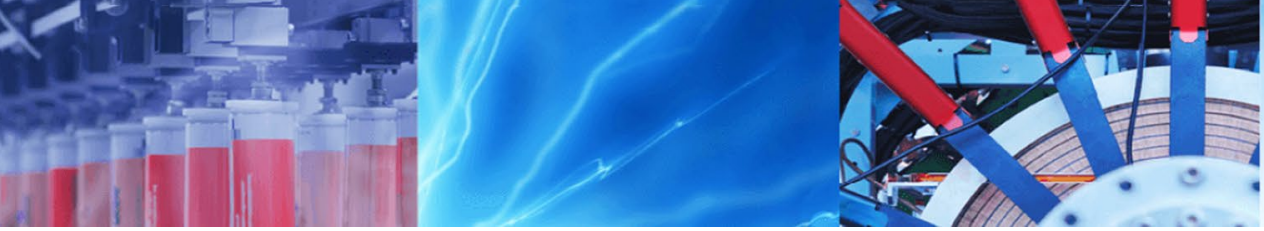

Research Article

\title{
Benzophenone based photostable fluorescent monoazo disperse dyes: Synthesis, AIE, viscosity, UPF and TD-DFT study
}

\author{
Suvidha S. Shinde ${ }^{1} \cdot$ Amol G. Jadhav $^{1} \cdot$ Nagaiyan Sekar $^{1}$ (I)
}

Received: 22 August 2020 / Accepted: 16 October 2020 / Published online: 2 December 2020

(c) Springer Nature Switzerland AG 2020

\begin{abstract}
Synthesis of a parallel series of hydrophobic azo dyes (4-hydroxy benzophenone and corresponding 4-methoxy benzophenone series) and a comprehensive systematic spectroscopic study in solvents of varying polarity and viscosity is presented. This includes glycerol-dimethylsulfoxide, toluene-polyethylene glycol 400 and toluene-paraffin oil viscous mixtures. Emission intensities of dyes (hydrazone forms) were enhanced by 47-96 folds in viscous solvent media compared to the non-viscous media. So, role of hydrogen bonding in the emission enhancement were verified for 4-hydroxy benzophenone series with 4-methoxy benzophenone series. Polarity alteration effect on spectroscopic properties of dyes in various viscous combinations has been correlated by solvent polarity graphs. Density Functional Theory (DFT), Time Dependent Density Functional Theory (TD-DFT), HOMO-LUMO energy gap and Electrophilicity-index estimations are in good contract with the experimental interpretations. Dyes on PET fibers showed excellent light fastness. Moreover, all the dyes on dyed fabrics exhibited good sublimation fastness and color values. Ultraviolet A/Ultraviolet B blocking and Ultraviolet Protection Factor (UPF) of all the dyes range 15-50 and are in good agreement with light fastness.
\end{abstract}

Keywords Benzophenone · Disperse dyes · Viscosity influenced emission · AIE · Electrophilicity index · UPF Factor and UVA/UVB blocking

\section{Introduction}

Azo dyes established as one of the leading dyestuff of industrial colorants [1]. Benzophenone is a well-known aromatic ketone obtained by organic synthesis or from natural products [2]. Benzophenone based compounds have wide applications as excellent UV absorbers [3] and thermal stabilizers [4]. Benzophenone based textile dyes are also well reported [5-7]. To attain the excellent photostability and hence light fastness property different approaches have been made [7-9]. In some cases, fabric dyed with commercially available disperse dyes disperse yellow 86 [10], Disperse Yellow GLFS [11], CI
Disperse Yellow 42 [12], 2,4-dihydroxy benzophenone and 4-hydroxy benzophenone were directly applied as UV absorber in the dyeing liquid or by conducting treatment to the dyed fabrics after dyeing [13]. Freeman et al. was the first to investigate the consequence of incorporation of photo-stabilizer moiety into Disperse Yellow 42 in 1992 [14]. Further lot of efforts have been focussed to increase light fastness of dyes by incorporating photo stabilising unit $[7,15,16]$. Also enhancement of the light fastness value is considered to be an important criteria in the textile industry, since Polyethylene Terephthalate (PET) fabrics are extensively used in coloured automobile stuffing, which can be visible to direct sunlight above $50^{\circ} \mathrm{C}$

Electronic supplementary material The online version of this article (https://doi.org/10.1007/s42452-020-03732-9) contains supplementary material, which is available to authorized users.

Nagaiyan Sekar, nethi.sekar@gmail.com; n.sekar@ictmumbai.edu.in | ${ }^{1}$ Department of Dyestuff Technology, Institute of Chemical Technology, Nathalal Parekh Marg, Matunga, Mumbai 400 019, India. 
[17]. In order to minimize the above said problems, textiles can be served to create sunblind sun umbrella and other shielding clothing. Compared to cosmetics, clothing can be considered as relatively appropriate barrier against UV radiations [18]. So, there is a need to design light fast fluorescent disperse dyes for light energy dissipations $[19,20]$.

Fluorescent dyes are having great significance in the colouring of synthetic fabrics (e.g. polyacrylonitrile, polyester and polyamides) [19]. Some of the important fluorescent textile dyes (usually disperse dyes) were applied on PET and thus can be considered as important disperse class of dyes [20]. Most of the commercial dyes are yellow, providing the very similar greenish-yellow fluorescent colour which allowed the dyed materials to be more bright, visible and simply noticeable and thus, very commonly encountered on garments worn for safety purposes [21]. The most important fluorescent disperse dyes are C.I. Disperse Yellows 82,184,186 and 232 and C.I. Disperse reds 277,374 . The commercially available fluorescent dyes for textile application are frequently objected for light fastness stability and most of them are incompatible with standards essential for the additional demanding applications [22].

It is reported in the literature that benzophenone and some of it's derivatives are emissive in nature [23-26]. Recently, Barsotti et al. have done wide-ranging work on4hydroxy benzophenone for it's fluorescence properties [23, 27]. In general emission properties of azobenzene dyes were induced or altered by various ways which include restricted isomerisation and rotations across $-\mathrm{N}=\mathrm{N}$ - group by N-B bond formation [28], by incorporation of benzothiazole fluorescent moiety to the azo dyes [29, 30], by cooling of dye sample to very low temperature or by embedding dyes in rigid solvents [31]. However, there are very few fluorescent azobenzenes reported so far [28, 32-37]. Considering decent $\pi$-conjugation [38] and restricted rotations across $-\mathrm{N}=\mathrm{N}-$ group [28], these benzophenone dyes can perform as good applicants for preparation of attractive photo stable fluorescent compounds.

Recently, effect of viscosity on fluctuating emission properties of some of the organic dyes were provided and studied in the literature $[39,40]$, however, only few reports were based on viscosity effects on the properties of azo dyes [20,41,42]. Intramolecular rotation in azo dyes are restricted due to either chemical interaction or adhesion of dyes on fabrics during dyeing $[43,44]$. So, it is important to consider intramolecular rotation contributed to the reduction mechanism for the dye in the solution [43]. Although azo-dyes are considered to be non-fluorescent specifically in solutions, but few of them became fluorescent when bound to the substrate [31]. Some of the monoazo dyes can act as molecular rotors, where fluorescence emission property is sensitive to solution [45]. Some of the azo dyes were reported with respect to emission intensity enhancement as viscosity of the solution increases [45]. This varying emission characteristics of azo dyes might be described on the basis of constrained rotational motion across the bonds by viscosity in the micro-environment around the molecule [45]. Briefly, it can be suggested that the either viscous micro-environment or the substrate (e.g. fibers)might be responsible for the generation of necessary restriction in azo dyes rotational motions which further contribute to overall emission of these dyes. So, it is of interest to study systematically viscous micro-environmental effects on emission properties of the benzophenone based mono azo disperse dyes.

To meet the requirements of fluorescent disperse dyes with excellent fastness properties, we used the key ingredient benzophenone which acts as a UV stabilizer and it has its own emission, which facilitate to prepare photostable fluorescent dyes. As azo $(-\mathrm{N}=\mathrm{N}-)$ moiety in azo dyes reduce the fluorescent properties because of photoisomerisation $[46,47]$ and other dynamics such as excimer or exciplex formation may be contribute to reduce fluorescence $[39,48]$. So, to avoid these process and increase fluorescence one can use micro-viscosity as a strategy to make molecule more rigid and restrict its rotations.

Here, we has synthesized a series of benzophenone inbuilt mono azo disperse dyes. Moreover, parallel series of methylated analogues enabled to restricted azohydrazone tautomerization to ensure study of exclusive azo form systematically. Detailed photophysical characterizations were performed to study effect of viscosity, polarity and role of hydrogen bonding in the emission enhancement in these dyes. The dyes are fully characterized in terms of their dyeing properties including the color assessment, sublimation fastness, and light fastness was carried out. Theoretical calculation by DFT supports our experimental outcomes.

\section{Experimental section}

\subsection{Materials and methods}

Sodium nitrite, sodium carbonate, sodium hydroxide, aniline, $p$-chloro aniline, $p$-nitro aniline, conc. $\mathrm{H}_{2} \mathrm{SO}_{4}$, conc. $\mathrm{HCl}$, urea, 4-hydroxy benzophenone, anhydrous methanol, acetone, dimethyl sulphate (DMS), N,N-dimethyl formamide (DMF), Saragen 50 (dispersing agent), sulphamic acid, sodium acetate, sodium chloride and acetic acid procured from Sigma Aldrich India. All A.R. mark organic solvents were procured from SD Fine Chemicals, Mumbai. All organic solvents after distillation as per the standard procedures were utilized further. Melting and boiling points of all the chemicals confirmed and used without any 
purifications. Ready for dyeing polyester (PET) (100\%) fabric (weight $70 \mathrm{~g} / \mathrm{m}^{2}$, Ends/105 in. and picks $/ 94$ in.), nylon (NYL) $(70 \mathrm{~d} / 25 \mathrm{f}$, plain weaved, $219 \times 191 / 5 \mathrm{~cm})$, acrylic (ACR) was purchased from Piyush Syndicate, Mumbai. For dyeing application Flexi dyer machine procured from Rossari Labtech Mumbai, India has been used.

Thin layer chromatography on $0.25 \mathrm{~mm}$ (silica gel 60F254 pre-coated aluminum plate) used to check completion of the reactions. Purification of all synthesized compounds accomplished using re-crystallizations technique. Melting point instrument procured from Veego Instruments, Mumbai, India. ${ }^{1} \mathrm{H}$-NMR and ${ }^{13} \mathrm{C}-\mathrm{NMR}$ spectra were documented on Agilent NMR $500 \mathrm{MHz}$ and $125 \mathrm{MHz}$ respectively. Chemical shifts stated in $\delta$-ppm and used $\mathrm{CDCl}_{3}$ as a solvent and TrimethylSilane (TMS) used as an internal standard. FT-IR spectra noted on JASCO-4100 FT-IR instrument with ATR accessories.

\subsection{Photophysical study}

Absorption Study of all synthesized dyes documented using Perkin Elmer UV-Vis spectrometer Lambda-25 instrument. Eight unlike polarity solvents used to make 1-5 $\mu \mathrm{M}$ concentration solutions of all dyes (e.g. toluene, 1,4 dioxane, chloroform $\left(\mathrm{CHCl}_{3}\right)$,ethyl acetate(EtOAc), methanol $(\mathrm{MeOH})$, tetrahydrofuran (THF), dimethylformamide (DMF) and dimethylsulphoxide (DMSO).

\subsection{Viscosity study and calculations}

Viscosity sensitivity study was carried out in various polarity viscous mixture such as DMSO-Glycerol, toluenepolyethyleneglycol400 (PEG-400) and toluene-paraffin oil mixture. Varian-Cary Eclipse fluorescence-spectrometer were used to document fluorescence emission spectra of all dyes for this constant concentration 1-5 $\mu \mathrm{M}$ solutions of dyes used. Viscosity $(\eta)$ of three viscous mixtures at different percentage ratio were mathematically estimated by equation [49] (Table S1).

$\ln \eta=X 1 \ln \eta 1+X 2 \ln \eta 2$

Where,

$\eta=$ viscosity

$\mathrm{X} 1, \mathrm{X} 2$ = mole fractions of binary mixture (for each constituent separately)

$\eta 1, \eta 2=$ viscosities of the binary mixture (for each constituent separately)

Varying dynamic viscosity in $\mathrm{mPas}^{*}$ against varying solvent ratios in volume $\%$ was plotted for three different binary mixtures (i.e. DMSO-Glycerol, toluene-PEG400 and toluene-paraffin oil) (Fig. S1).

\subsection{Computational study}

DFT computational experiments executed on a HP-workstation XW-8600 with4GB RAM, Xeon processor and Windows Vista as the operating systems. Gaussian $09 \mathrm{~W}$ used as the software package [50]. Geometry of ground state optimized by using B3LYP as a functional and 6-311G (d) as a basis set for all the molecules [51, 52]. Oscillator strengths, Vertical excitation energies and orbital contributions (lowest 10 singlet transitions) to the optimized geometry of the ground states of molecules were attained by TD-DFT computations. Polarizable Continuum Model (PCM) implemented for optimizations in various solvent phases in Gaussian 09 [53]. The solvents used in DFT study were toluene, 1,4-dioxane, $\mathrm{CHCl}_{3}$, EtOAc, $\mathrm{MeOH}$, THF, DMF and DMSO.

\subsection{Dyeing study}

\subsubsection{Color assessment}

Color assessment the dyed substrate done on Spectra Scan-5100+ with $10^{\circ}$ standard observer under D65illuminant.K/S value of dyed substrate determined by Kubelka-Munk equation.

\subsubsection{Fastness property}

Dyed samples were tested for light fastness by Q-Sun Xenon Test Chamber (Q-Lab Corporation, Ohio, USA)by AATCC 16/2004 method.

Sublimation fastness tester, RBE Electronics Engineering Private Limited Mumbai, India has been used to evaluate sublimation property of dyed sample by ISO 105-PO1 1993standard method [54].

The change in the shade along with stainings of the in-line fabrics, were evaluated according SDC (Society of Dyers \& Colorists) grey scale.

\subsubsection{Percentage exhaustion determination}

Exhaustion (\%) of the of the dye bath were estimated by method [55].

\subsubsection{UPF Factor determination}

UPF and UVA and UVB percentage transmission estimated by AATCC Test Method-183.Blocking or Transmittance of Erythemally Weighted UV (Ultra-violet)radiations through fabric by AATCC, 2002 (AATCC - Technical Manual, 2015). UV transmittance analyzer was used for the measurements for UPF testing. Measurements were performed using a 
UV transmittance UPF Tester V-2000F Fabric Analyzer, Labsphere, US.

\subsection{Syntheses and characterization}

The synthetic skeleton of synthesized azo dyes (4a-4c) and $(\mathbf{5 a - 5 c )}$ is shown in Scheme 1. Dye $\mathbf{4 c}$ is reported in the literature [56].

\subsubsection{Synthesis of azo dyes (4a-4c)}

The mixture having aromatic amines $(0.003 \mathrm{~mol})$, conc. Hydrochloric acid $(\mathrm{HCl}) 3 \mathrm{ml}(0.035 \mathrm{~mol})$, and $15 \mathrm{ml}$ $(1.38 \mathrm{~mol})$ water heated till solution become cleared and this mixture cooled down to $0^{\circ} \mathrm{C}$. The acidity range of the solution determined by using litmus paper. Sodium nitrite $0.25 \mathrm{~g}(0.003 \mathrm{~mol})$ solution was little by little added in step wise manner with constant stirring at below $5^{\circ} \mathrm{C}$ temperature. Further solution permitted to stir for $40 \mathrm{~min}$ at $5^{\circ} \mathrm{C}$, after complete addition. Excess nitrous acid quenched by Urea. Meanwhile, sodium hydroxide $(0.025 \mathrm{~N}, 0.003 \mathrm{~mol})$ was dissolved the solution containing 4-hydroxy benzophenone $(0.5 \mathrm{~g}, 0.003 \mathrm{~mol})$ as coupler resulting into clear solution ( $\mathrm{pH}=11-12)$. The above diazonium mixture slowly added to the coupler solution with continuous stirring at temperature $0-5{ }^{\circ} \mathrm{C}$. While addition of diazonium salt to the coupler solution $\mathrm{pH}$ decreases, so $\mathrm{Na}_{2} \mathrm{CO}_{3}(10 \%$ $w / v)$ solution was slowly added to the reaction medium to avoid further decrease in $\mathrm{pH}$ and was maintain in between 7.5-8.0 [6]. The further mixture was stirred for $3 \mathrm{~h}$ (Scheme 1). The precipitated resultant azo dye filtered and thoroughly washed with water. Recrystallization in ethanol gave desired azo compound. Yield of $\mathbf{4 a - 4 c}$ after recrystallization was then reported.

\subsubsection{Characterizations}

4a: Pale yellow powder, Yield-75\%, melting point: $130-132^{\circ} \mathrm{C}$.

${ }^{1} \mathbf{H}-\mathrm{NMR} \boldsymbol{\delta}_{\mathbf{H}}\left(\mathbf{5 0 0} \mathbf{~ M H z}, \mathrm{CDCl}_{\mathbf{3}}\right): 13.48(1 \mathrm{H}, \mathrm{s},-\mathrm{OH})$, 8.50-8.45 (1H, m, Ar-H), 7.98-7.94 (1H, m, Ar-H), 7.92-7.88 $(2 \mathrm{H}, \mathrm{m}, \mathrm{Ar}-\mathrm{H}), 7.84(2 \mathrm{H}, \mathrm{d}, J=8 \mathrm{~Hz}, \mathrm{Ar}-\mathrm{H}), 7.66-7.60(1 \mathrm{H}$, $\mathrm{m}, \operatorname{Ar}-\mathrm{H}), 7.55(2 \mathrm{H}, \mathrm{d}, J=1 \mathrm{~Hz}, \operatorname{Ar}-\mathrm{H}), 7.53(2 \mathrm{H}, \mathrm{s}, \operatorname{Ar}-\mathrm{H})$, 7.53-7.51 $(1 \mathrm{H}, \mathrm{m}, \mathrm{Ar}-\mathrm{H}), 7.15(1 \mathrm{H}, \mathrm{dd}, J=8 \mathrm{~Hz}$ and $1 \mathrm{~Hz}$, Ar-H) ppm.

${ }^{13} \mathrm{C}-\mathrm{NMR} \boldsymbol{\delta}_{\mathrm{C}}\left(\mathbf{1 2 5} \mathbf{M H z}, \mathrm{CDCl}_{3}\right):$ 194.7, 156.7, 150.0, $137.8,136.4,136.0,134.9,132.2,131.8,129.7,129.6,129.4$, $128.4,122.4,118.6 \mathrm{ppm}$.

FT-IR: 3057 (phenolic OH), $1680(\mathrm{C}=\mathrm{O}), 1644(\mathrm{C}=\mathrm{C}), 1275$ $(\mathrm{C}-\mathrm{N}) \mathrm{cm}^{-1}$.

HRMS: $303.1189\left(\mathrm{M}+\mathrm{H}^{+}\right)\left(\right.$Calculated for $\mathrm{C}_{19} \mathrm{H}_{15} \mathrm{~N}_{2} \mathrm{O}_{2}$ : 303.1165).

4b: Pale yellow powder, Yield- $85 \%$, melting point: 204-206 ${ }^{\circ} \mathrm{C}$.

${ }^{1} \mathrm{H}-\mathrm{NMR} \boldsymbol{\delta}_{\mathrm{H}}\left(\mathbf{5 0 0} \mathbf{~ M H z}, \mathrm{CDCl}_{3}\right): 13.23(1 \mathrm{H}, \mathrm{s},-\mathrm{OH}), 8.45$ $(1 \mathrm{H}, \mathrm{d}, J=2 \mathrm{~Hz}, \mathrm{Ar}-\mathrm{H}), 7.96(1 \mathrm{H}, \mathrm{dd}, J=8.5 \mathrm{~Hz}$ and $2.0 \mathrm{~Hz}$, $\operatorname{Ar}-\mathrm{H}), 7.86(1 \mathrm{H}, \mathrm{s}, \mathrm{Ar}-\mathrm{H}), 7.84(1 \mathrm{H}, \mathrm{s}, \mathrm{Ar}-\mathrm{H}), 7.83(1 \mathrm{H}, \mathrm{s}$, Ar-H), 7.65-7.60 (2H, m, Ar-H), $7.53(1 \mathrm{H}, \mathrm{s}, \mathrm{Ar}-\mathrm{H}), 7.53(2 \mathrm{H}$, $\mathrm{d}, J=2 \mathrm{~Hz}, \operatorname{Ar}-\mathrm{H}), 7.51(1 \mathrm{H}, \mathrm{s}, \operatorname{Ar}-\mathrm{H}), 7.15(1 \mathrm{H}, \mathrm{d}, J=8.5 \mathrm{~Hz}$, Ar-H) ppm.

${ }^{13}$ C-NMR $\delta_{\mathrm{C}}\left(125 \mathrm{MHz}, \mathrm{CDCl}_{3}\right):$ 194.6, 156.6, 148.5, 137.8, 137.7, 136.4, 136.0, 135.1, 132.2, 129.8, 129.8, 129.7, $128.4,123.6,118.6 \mathrm{ppm}$.
Scheme 1 Synthetic scheme of mono azo disperse dyes $(4 a-4 c$ and $5 a-5 c)$

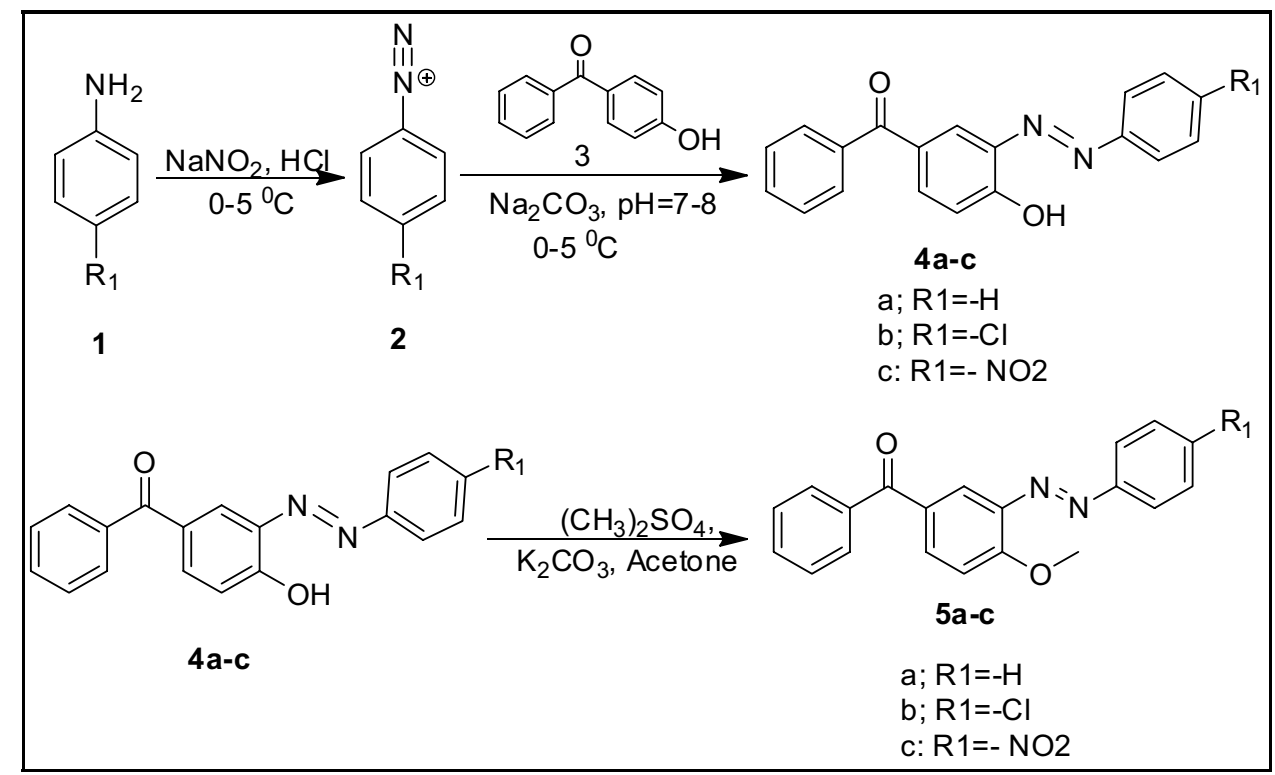


FT-IR: 3063 (phenolic OH), 1691 ( $C=0), 1652$ ( $C=C), 1284$ $(\mathrm{C}-\mathrm{N}), 620(\mathrm{C}-\mathrm{Cl}) \mathrm{cm}^{-1}$.

HRMS: $335.0622\left(\mathrm{M}-\mathrm{H}^{+}\right)\left(\right.$Calculated for $\mathrm{C}_{19} \mathrm{H}_{12} \mathrm{CIN}_{2} \mathrm{O}_{2}$ : 335.0619).

4c: Pale yellow powder, Yield- $85 \%$, melting point: 194-195 ${ }^{\circ} \mathrm{C}$.

${ }^{1} \mathbf{H}-\mathbf{N M R} \boldsymbol{\delta}_{\mathrm{H}}\left(\mathbf{5 0 0} \mathbf{~ M H z}, \mathbf{C D C l}_{3}\right): 13.04(1 \mathrm{H}, \mathrm{s},-\mathrm{OH}), 8.51$ $(1 \mathrm{H}, \mathrm{s}, \mathrm{Ar}-\mathrm{H}), 8.41(2 \mathrm{H}, \mathrm{d}, J=8.5 \mathrm{~Hz}, \mathrm{Ar}-\mathrm{H}), 8.05(1 \mathrm{H}, \mathrm{s}, \mathrm{Ar}-\mathrm{H})$, $8.02(2 \mathrm{H}, \mathrm{d}, J=9 \mathrm{~Hz}, \mathrm{Ar}-\mathrm{H}), 7.84(2 \mathrm{H}, \mathrm{d}, J=7.5 \mathrm{~Hz}, \mathrm{Ar}-\mathrm{H}), 7.64$ $(1 \mathrm{H}, \mathrm{t}, J=7.5 \mathrm{~Hz}, \mathrm{Ar}-\mathrm{H}), 7.54(2 \mathrm{H}, \mathrm{t}, J=7.5 \mathrm{~Hz}, \mathrm{Ar}-\mathrm{H}), 7.18(1 \mathrm{H}$, $\mathrm{d}, J=8.5 \mathrm{~Hz}$, Ar-H) ppm.

${ }^{13}$ C-NMR $\delta_{\mathrm{C}}\left(125 \mathrm{MHz}, \mathrm{CDCl}_{3}\right): 194.3,156.5,153.5$, $149.0,137.5,137.1,136.4,132.8,132.4,130.2,129.7,128.4$, $125.0,123.0,118.9 \mathrm{ppm}$.

FT-IR: 3049 (phenolic OH), $1673(\mathrm{C}=\mathrm{O}), 1638(\mathrm{C}=\mathrm{C}), 1272$ $(\mathrm{C}-\mathrm{N}) \mathrm{cm}^{-1}$.

HRMS: $346.0848\left(\mathrm{M}-\mathrm{H}^{+}\right)$(Calculated for $\mathrm{C}_{19} \mathrm{H}_{12} \mathrm{~N}_{3} \mathrm{O}_{2}$ : 346.0868).

\subsubsection{Preparation of methylated forms of azo dyes $(\mathbf{5 a}, \mathbf{5} \mathbf{b}$,} 5c)

General procedure for preparation of azo dyes (5a-5c) Dye $\mathbf{4 a}, \mathbf{4 b}$, or $\mathbf{4 c}$ was dissolved in acetone along with potassium carbonate ( 3 eq.) and DMS ( 0.5 eq.) in $250 \mathrm{ml}$ round bottom flask. The reaction mass was allowed to stirred at $25^{\circ} \mathrm{C}$ for $30 \mathrm{~min}$. Reaction was monitored by silica gel TLC. After the starting was consumed, acetone was concentrated under reduce pressure on rota evaporator and solid form thoroughly washed with water and dried. The obtained dyes was pure and single spotted on TLC further no purification is carried out. Therefore, reported yield of $\mathbf{5 a}-\mathbf{c}$ is in its pure form with very good yields.

\subsubsection{Characterizations}

5a: Yellow powder, Yield- $96 \%$, melting point: $204^{\circ} \mathrm{C}$.

${ }^{1} \mathbf{H}-\mathrm{NMR} \boldsymbol{\delta}_{\mathbf{H}}\left(\mathbf{5 0 0} \mathbf{~ M H z}, \mathrm{CDCl}_{\mathbf{3}}\right): 8.12(1 \mathrm{H}, \mathrm{d}, J=2 \mathrm{~Hz}$, Ar-H), $7.99(1 \mathrm{H}, \mathrm{dd}, J=8.5,2 \mathrm{~Hz}, \mathrm{Ar}-\mathrm{H}), 7.92(1 \mathrm{H}, \mathrm{s}, \mathrm{Ar}-\mathrm{H})$, $7.91(1 \mathrm{H}, \mathrm{s}, \mathrm{Ar}-\mathrm{H}), 7.82(1 \mathrm{H}, \mathrm{s}, \mathrm{Ar}-\mathrm{H}), 7.80(1 \mathrm{H}, \mathrm{s}, \mathrm{Ar}-\mathrm{H}), 7.60$ $(1 \mathrm{H}, \mathrm{t}, J=7.5 \mathrm{~Hz}, \mathrm{Ar}-\mathrm{H}), 7.54-7.48(5 \mathrm{H}, \mathrm{m}, \mathrm{Ar}-\mathrm{H}), 7.19(1 \mathrm{H}, \mathrm{d}$, $J=8.5 \mathrm{~Hz}, \mathrm{Ar}-\mathrm{H}), 4.12\left(3 \mathrm{H}, \mathrm{s},-\mathrm{OCH}_{3}\right) \mathrm{ppm}$.

${ }^{13} \mathrm{C}-\mathrm{NMR} \boldsymbol{\delta}_{\mathrm{C}}\left(125 \mathrm{MHz}, \mathrm{CDCl}_{3}\right): 195.2,159.9,152.9$, $141.6,137.8,134.2,132.2,131.2,130.1,129.8,129.1,128.3$, $123.1,119.4,112.1,56.5 \mathrm{ppm}$.

FT-IR: $1688(\mathrm{C}=\mathrm{O}), 1651(\mathrm{C}=\mathrm{C}), 1274(\mathrm{C}-\mathrm{N}), 1013$ $\left(\mathrm{O}-\mathrm{CH}_{3}\right) \mathrm{cm}^{-1}$.

HRMS: $317.1353\left(\mathrm{M}+\mathrm{H}^{+}\right)\left(\right.$Calculated for $\mathrm{C}_{20} \mathrm{H}_{17} \mathrm{~N}_{2} \mathrm{O}_{2}$ : 317.1374).

5b: Yellow powder, Yield $-92 \%$, melting point: $163-165^{\circ} \mathrm{C}$.

${ }^{1} \mathbf{H}-\mathbf{N M R} \boldsymbol{\delta}_{\mathbf{H}}\left(500 \mathbf{~ M H z}, \mathrm{CDCl}_{3}\right): 8.12(1 \mathrm{H}, \mathrm{s}, \mathrm{Ar}-\mathrm{H}), 8.00$ $(1 \mathrm{H}, \mathrm{d}, J=9 \mathrm{~Hz}, \operatorname{Ar}-\mathrm{H}), 7.87(2 \mathrm{H}, \mathrm{d}, J=8.5 \mathrm{~Hz}, \mathrm{Ar}-\mathrm{H}), 7.80$
$(2 \mathrm{H}, \mathrm{d}, J=8 \mathrm{~Hz}, \mathrm{Ar}-\mathrm{H}), 7.61(1 \mathrm{H}, \mathrm{t}, J=8 \mathrm{~Hz}, \mathrm{Ar}-\mathrm{H}), 7.51(2 \mathrm{H}$, $\mathrm{d}, J=8 \mathrm{~Hz}, \operatorname{Ar}-\mathrm{H}), 7.48(2 \mathrm{H}, \mathrm{d}, J=8.5 \mathrm{~Hz}, \operatorname{Ar}-\mathrm{H}), 7.19(1 \mathrm{H}, \mathrm{d}$, $J=8.5 \mathrm{~Hz}, \mathrm{Ar}-\mathrm{H}), 4.12\left(3 \mathrm{H}, \mathrm{s},-\mathrm{OCH}_{3}\right) \mathrm{ppm}$.

${ }^{13}$ C-NMR $\delta_{\mathrm{C}}$ (125 MHz, CDCl3): 195.2, 16.1, 151.2, 141.4, 137.7, 137.1, 134.5, 132.2, 130.1, 129.8, 129.3, 128.3, 124.3, $119.3,112.2,56.6 \mathrm{ppm}$.

FT-IR: $1694(\mathrm{C}=\mathrm{O}), 1661(\mathrm{C}=\mathrm{C}), 1283(\mathrm{C}-\mathrm{N}), 1008\left(\mathrm{O}-\mathrm{CH}_{3}\right)$ $\mathrm{cm}^{-1}$.

HRMS: $351.0955\left(\mathrm{M}+\mathrm{H}^{+}\right)\left(\right.$Calculated for $\mathrm{C}_{20} \mathrm{H}_{16} \mathrm{CIN}_{2} \mathrm{O}_{2}$ : 351.0965).

5c: Yellow powder, Yield $-88 \%$, melting point: $225^{\circ} \mathrm{C}$.

${ }^{1} \mathbf{H}-N M R \boldsymbol{\delta}_{\mathbf{H}}\left(\mathbf{5 0 0} \mathbf{~ M H z}, \mathrm{CDCl}_{\mathbf{3}}\right): 8.39(1 \mathrm{H}, \mathrm{s}, \mathrm{Ar}-\mathrm{H}), 8.37$ $(1 \mathrm{H}, \mathrm{s}, \mathrm{Ar}-\mathrm{H}), 8.18(1 \mathrm{H}, \mathrm{d}, J=2 \mathrm{~Hz}, \mathrm{Ar}-\mathrm{H}), 8.06(1 \mathrm{H}, \mathrm{dd}$, $J=8.5 \mathrm{~Hz}$ and $2 \mathrm{~Hz}, \operatorname{Ar}-\mathrm{H}), 8.04(1 \mathrm{H}, \mathrm{s}, \operatorname{Ar}-\mathrm{H}), 8.02(1 \mathrm{H}, \mathrm{s}$, Ar-H), $7.81(2 \mathrm{H}, \mathrm{d}, J=8.5 \mathrm{~Hz}, \operatorname{Ar}-\mathrm{H}), 7.62(1 \mathrm{H}, \mathrm{t}, J=7.5 \mathrm{~Hz}$, Ar-H), $7.52(2 \mathrm{H}, \mathrm{t}, J=7.5 \mathrm{~Hz}, \operatorname{Ar}-\mathrm{H}), 7.23(1 \mathrm{H}, \mathrm{d}, J=8.5 \mathrm{~Hz}$, Ar-H), $4.15\left(3 \mathrm{H}, \mathrm{s},-\mathrm{OCH}_{3}\right)$ ppm.

${ }^{13}$ C-NMR $\delta_{\mathrm{C}}(125 \mathrm{MHz}, \mathrm{CDCl}): 195.0,160.6,155.9$, $148.8,141.3,137.6,135.6,132.4,131.1,129.9,128.4,124.7$, 123.6, 119.2, 112.4, $56.7 \mathrm{ppm}$.

FT-IR: $1685(\mathrm{C}=\mathrm{O}), 1649(\mathrm{C}=\mathrm{C}), 1273(\mathrm{C}-\mathrm{N}), 1105\left(\mathrm{O}-\mathrm{CH}_{3}\right)$ $\mathrm{cm}^{-1}$.

HRMS: $362.1137\left(\mathrm{M}+\mathrm{H}^{+}\right)\left(\right.$Calculated for $\mathrm{C}_{20} \mathrm{H}_{16} \mathrm{~N}_{3} \mathrm{O}_{2}$ : 362.1161).

\subsubsection{Procedure of dyeing (PET, Nylon, Acrylic)}

PET, nylon and acrylic fabrics dyeing were performed on a Flexi Dyer dyeing machine with 1:30 MLR (material to liquor ratio) and percentage shade $2 \%$ (calculated on the weight of fabric). Firstly, the azo dye was pasted using dispersing agent Saragen-50 and further diluted using $15 \mathrm{ml}$ of buffered solution with acetic acid in water ( $\mathrm{pH} 4-5)$ [57]. Complete dispersion of the dye in water was acquired by ultra-sonication for 30 min. PET, NYL and ACR substrates dyed using above solutions. Dyeing was initiated at room temperature and raised to $130^{\circ} \mathrm{C}(\mathrm{PET})$ and $90^{\circ} \mathrm{C}$ (nylon), $90{ }^{\circ} \mathrm{C}$ (acrylic) respectively, maintain this raised temperature for $1 \mathrm{~h}$ and cool down to $60^{\circ} \mathrm{C}$. The dyed substrate thoroughly washed with warm and cold water and further for PET dyed fabric, reduction clearing treatment is given with $2 \mathrm{gpl}$ soda ash $\left(\mathrm{Na}_{2} \mathrm{CO}_{3}\right), 1 \mathrm{gpl}$ soap solution and $2 \mathrm{gpl}$ Sodium hydrosulphite at $70{ }^{\circ} \mathrm{C}$ for $15 \mathrm{~min}$, then treated fabric was washed with water and dried.

\section{Results and discussion}

\subsection{Photo-physical properties}

Dyes (4a-4c) showed dual absorption peaks while their corresponding methoxy derivatives $(\mathbf{5 a}-\mathbf{5 c}$ ) showed only single absorption peak (Fig. S2). Significant effect 
of substituents ( $-\mathrm{Cl}$ and $-\mathrm{NO}_{2}$ group) was observed on absorption maxima of dyes (4b and $\mathbf{4} \mathbf{c}$ ) on comparing with unsubstitued dye (4a) in various solvents. But on comparing $\mathbf{4 b}(-\mathrm{Cl})$ with $\mathbf{4 c}\left(-\mathrm{NO}_{2}\right)$, a very small difference was detected in the absorption maxima of the dyes. Only $\mathbf{4 b}$ dye is slightly red shifted (1-3 nm) compared to $4 \mathrm{c}$ in different solvents. Effect of $-\mathrm{NO}_{2}$ and $-\mathrm{Cl}$ substituents on absorption spectra of the dyes are provided in the literature $[58,59]$. The first absorption maxima of $\mathbf{4 a - 4 c}$ corresponds to the azo form, while the second red shifted absorption maxima corresponds to the hydrazone form [60]. Methoxy group is better donating group as compared to the hydroxyl group and significant effect has been observed in absorption spectra. $\mathbf{5 a}, \mathbf{5} \mathbf{b}$ and $\mathbf{5 c}$ (exclusive azo forms) are absorbing at red shifted region as compared to corresponding azo form of hydroxyl derivatives $\mathbf{4 a}, \mathbf{4} \mathbf{b}$ and $\mathbf{4} \mathbf{c}$, respectively. Additionally, distinct second absorption peak (red shifted) of hydrazone form at around 369-382 nm was observed for compounds $\mathbf{4 a}, \mathbf{4 b}$ and $\mathbf{4 c}$ which was disappeared in methoxy series $(\mathbf{5 a}, \mathbf{5 b}$ and $\mathbf{5 c})$ due to the absence of hydrazone formation. Methoxy substituted parallel series $(\mathbf{5 a}, \mathbf{5 b}$ and $\mathbf{5 c})$ showed similar trend solvatochromism as compared to their corresponding hydroxyl analogues $(\mathbf{4 a}, \mathbf{4 b}$ and $\mathbf{4 c})$. The absorption maxima trend of dyes is as follows: $\mathbf{4 a}<\mathbf{4 c} \leq \mathbf{4 b}<\mathbf{5 a} \leq \mathbf{5 b}<\mathbf{5 c}$ (Table 1). It is clearly observed that dyes $\mathbf{4 a}, \mathbf{4 b}$ and $\mathbf{4 c}$ has very good molar absorptivity as matched to methoxy analogues $\mathbf{5 a}$, $\mathbf{5 b}$ and $\mathbf{5 c}$, respectively (Table 1 ).

\subsection{Viscosity influenced spectroscopic characterization}

Synthesized azo dyes are not emissive in solvents. The possible for non-radiative relaxation during de-excitation may be isomerizations and rotational motions through single bonds beside to azo group [61]. Substituent effects, solvent viscosity, solvent polarity and hydrogen bonding are also possible factors affects non-radiative rate [62-65]. So, viscosity helps to restrict rotations through the single bonds adjacent to azo. Azo-hydrazone tautomerism can also has contribution in restriction of the rotations in these dyes additionally. With the aim of consider polarity effects different polarity-viscosity binary mixtures (DMSO-Glycerol), (toluenePEG400) and (toluene-paraffin oil) have been used. In this study we have chosen three different viscous binary mixtures i.e. DMSO-Glycerol (polar protic), toluene-PEG 400 (polar aprotic) and toluene-paraffin oil (non-polar aprotic). Quinine sulphate (in $0.5 \mathrm{M} \mathrm{H}_{2} \mathrm{SO}_{4}, \Phi_{\mathrm{fl}}=0.546$ ) is used as a standard to calculate relative fluorescence quantum yield $\left(\Phi_{\mathrm{fl}}\right)$.

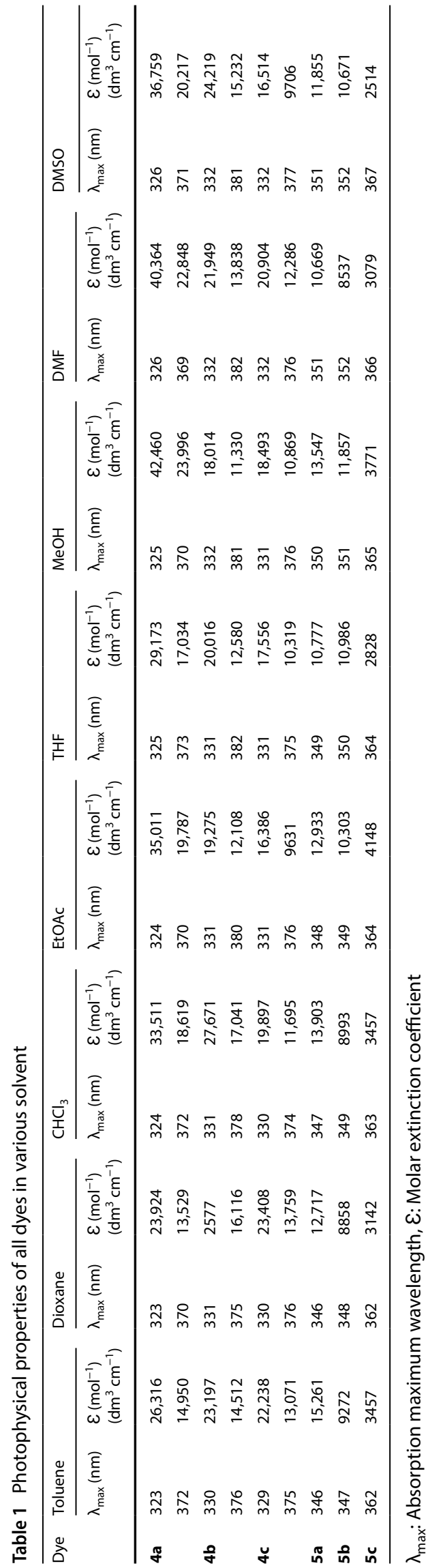




\subsubsection{DMSO-glycerol mixture}

\section{(a) Spectral data}

Absorption maxima of dyes were documented in varying extremely viscous medium Glycerol-DMSO at constant concentration (Fig. 1). Dyes (4a-4c) exhibited broad and dual absorption peaks (azo form and hydrazone form) while only single absorption peak with slight red shift was observed for the corresponding methoxy derivatives (5a-5c) (Fig. 1).

Azo forms of dyes (4aA, $\mathbf{4 b A}$ and $\mathbf{4} \mathbf{c A}$ ) did not exhibit noteworthy emission intensity progressing Glycerol-DMSO mixtures (Fig. 2). Calculated relative fluorescence quantum yields and fold of increase and at Glycerol(9.5):DMSO(0.5) v/v compared to blank DMSO for the dyes are 8.8 fold $\left(\Phi_{f l}=4 \%\right)$ for 4aA, 8.3 fold $\left(\Phi_{\mathrm{fl}}=5 \%\right)$ for $\mathbf{4 b A}$ and 6.3 fold $\left(\Phi_{\mathrm{fl}}=3 \%\right)$ for 4cA. Similar trends were observed for the corresponding methoxy analogues, i.e. 5a showed 17.5 fold $\left(\Phi_{f l}=3 \%\right)$, 5 b showed 15.7 fold $\left(\Phi_{f l}=3 \%\right)$ and 5c showed 12.5 fold $\left(\Phi_{f l}=4 \%\right)$ at Glycerol (9.5): DMSO(0.5) $v / v$ binary mixture (Fig. 2). On the other hand, hydrazone form of dyes $\mathbf{4 a H}, \mathbf{4 b H}$ and $\mathbf{4 c H}$ in Glycerol-DMSO binary mixture exhibited significant emission intensity enhancement with increasing Glycerol content. Hydrazone forms $\mathbf{4 a H}$ showed 96 fold $\left(\Phi_{\mathrm{fl}}=30 \%\right)$, $\mathbf{4 b H}$ showed 78 fold $\left(\Phi_{\mathrm{fl}}=27 \%\right)$ and 4cH showed 75 fold $\left(\Phi_{\mathrm{fl}}=29 \%\right)$ at Glycerol (9.5):DMSO (0.5) v/v (Fig. 2).
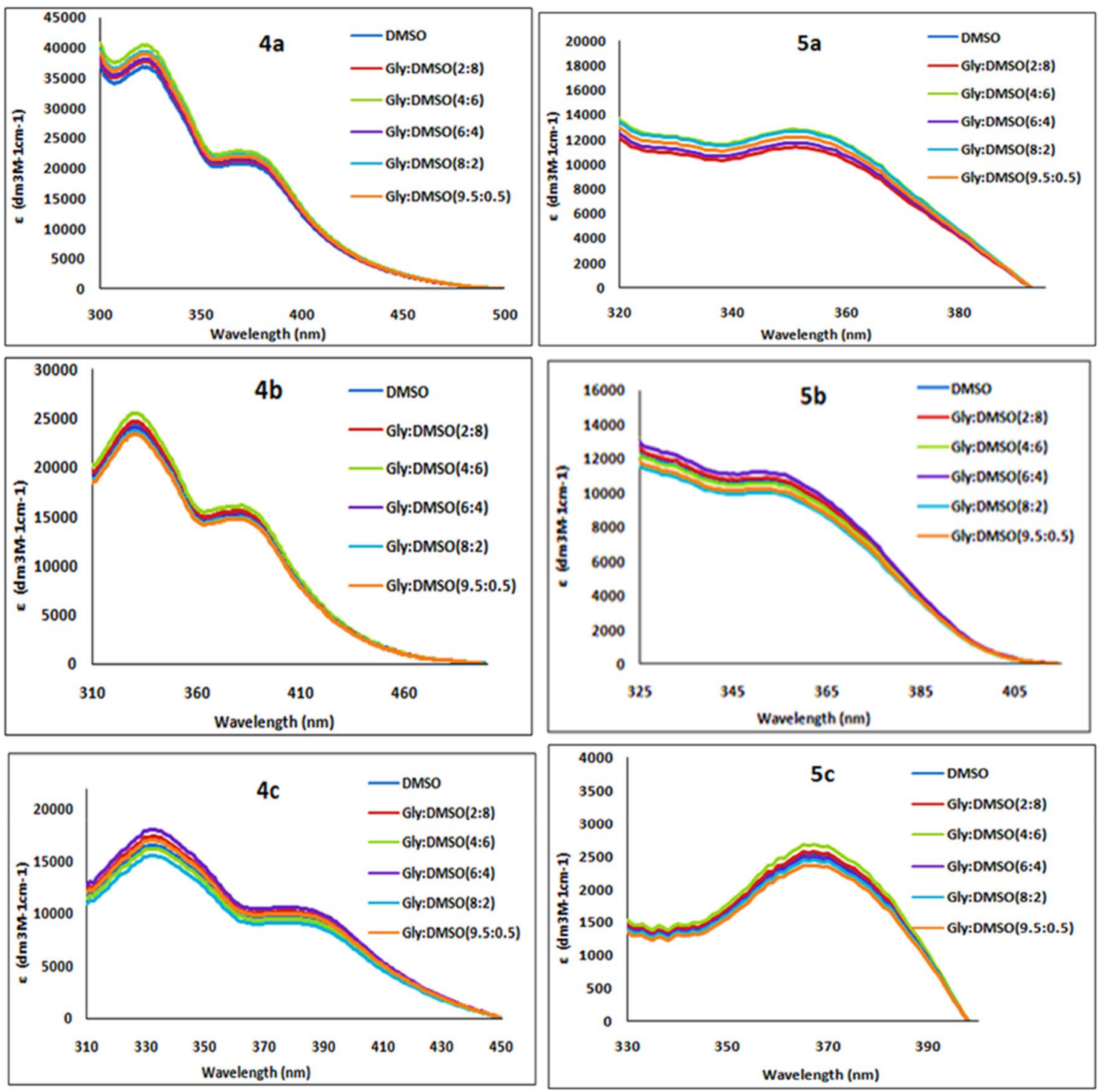

Fig. 1 Absorption spectra of $\mathbf{4 a}, \mathbf{4 b}, \mathbf{4 c}$ and $\mathbf{5 a}, \mathbf{5 b}, \mathbf{5 c}$ dyes in DMSO-Glycerol mixture ratio 

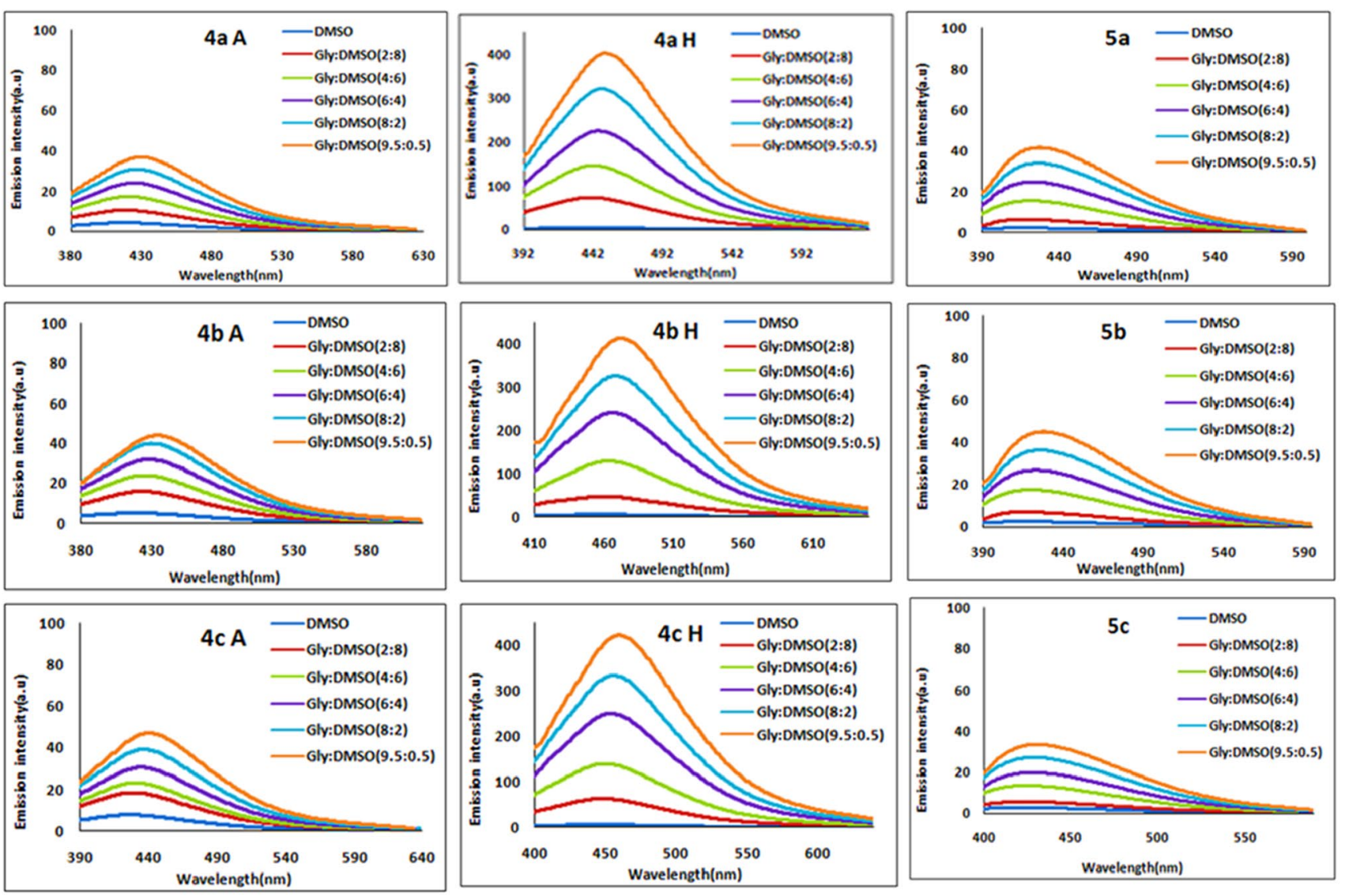

Fig. 2 Emission spectra of 4aA (excited @ 326 nm), 4bA (excited @ 333 nm) and 4cA (excited @ 332 nm), 5a (excited @ 351 nm), 5b (excited @ 365 nm), 5c(excited @ 367 nm), 4aH (excited @ 372 nm),

This result suggest hydrogen bonding involved in azo-hydrazone tautomerizm along with viscosity has a significant part in emission enhancement. Methoxy analogues of these dyes $\mathbf{5 a}, \mathbf{5} \mathbf{b}$ and $\mathbf{5 c}$ did not show significant emission intensity enhancement may be due to absence of hydrogen bonding center. Alternately, this can be explained and supported by more planarity attainment of hydrazone forms than the corresponding azo-methoxy forms of the dyes in viscous environment. Hence, both azo form and methoxy forms are less emissive as compared to hydrazone forms of the dyes.

(b) Viscosity sensitivity result

Forster-Hoffman equation is used to quantify viscosity sensitivity of synthesized azo dyes

$\log I=C+x \log$

Where,

$\eta=$ viscosity (mPa s).

$X=$ viscosity sensitivity parameter,

$I=$ maximum emission intensity and.
4bH (excited @ 384 nm) and 4cH (excited @ 381 nm) in DMSOGlycerol mixture ratio

\section{$C=$ constant.}

Viscosity sensitivity against emission intensity plotted. Viscosity rises from $1.99 \mathrm{mPa}$ (DMSO) to $688.51 \mathrm{mPa}$ s for (DMSO:Gly: 5:95).It is clearly observed that all the hydrazone forms of the dyes have significant viscosity sensitivity $4 \mathrm{aH}(\mathrm{x}=\mathbf{0 . 6 7 6})$, $4 \mathbf{b H}(x=0.690)$ and $4 \mathrm{cH}(x=0.673)$ with good regression coefficients on excitation @ 370-381 nm (Fig. 3). On the other hand azo forms $4 \mathrm{aA}, 4 \mathrm{bA}$ and $4 \mathrm{cA}$ have exhibited viscosity sensitivity $(x) \mathbf{0 . 3 4 9}, 0.337$ and $\mathbf{0 . 2 9 2}$, respectively. Similarly, methoxy analogues $\mathbf{5 a}, \mathbf{5 b}$ and $\mathbf{5 c}$ have exhibited viscosity sensitivity $(\mathbf{x})$ $0.499,0.456$ and $\mathbf{0 . 4 7 0}$, respectively. Comparative viscosity trend for all the dyes is found to be $\mathbf{4 b H} \geq \mathbf{4}$ $\mathbf{a H} \geq \mathbf{4 c H}>5 \mathrm{a} \geq \mathbf{5 c} \geq \mathbf{5 b}>\mathbf{4 a A} \geq \mathbf{4 b A} \geq \mathbf{4 c A}$ (Fig. 3).

(c) Viscosity polarity effect

As polarity of viscous media increases not only emission intensity enhancement but also red shifting in emission wavelength has been observed. Therefore, we have related solvent polarity functions with stokes shift and emission wavelength of dyes. For this purpose Lippert-Mataga and Weller equa- 


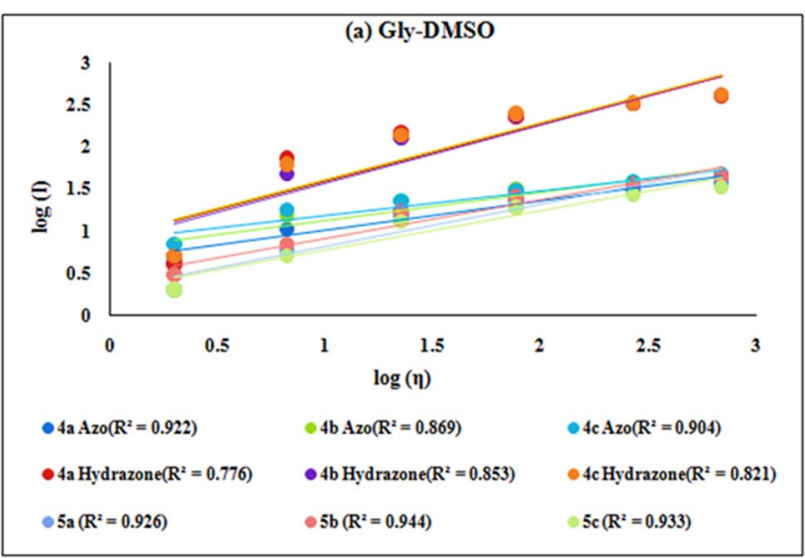

Fig. 3 Comparative viscosity against intensity (logarithmic) spectrum of dyes in different DMSO-Glycerol mixture ratio

tion has been used and solvatochromic behavior of synthesized dyes estimated. Lippert-Mataga plot indicated that azo forms of the dyes $\mathbf{4 a A}, \mathbf{4 b A}$ and $\mathbf{4 c A}$ exhibited large Stokes shifts $6646-7649 \mathrm{~cm}^{-1}$ and positive solvatochromism (Fig. 4). On the other hand small Stokes shifts and positive solvatochromism were observed for corresponding methoxy analogues $5 a, 5 b$ and $5 c\left(3828-5126 \mathrm{~cm}^{-1}\right)$. Similarly, hydrazone forms of the dyes showed small Stokes shift and positive solvatochromism $\left(4053-5015 \mathrm{~cm}^{-1}\right)$ and are in good correlation to fluorescence quantum yields.

Maximum Stokes shifts were observed for $\mathbf{4 a - 4 c}$ and found to have very good correlation of having low fluorescence quantum yields. Weller plot showed that azo forms of the dyes (4aA, $\mathbf{4 b A}$ and $\mathbf{4 c A})$ exhibited emission wavelengths $16,000-23,980 \mathrm{~cm}^{-1}$. Similar trend was observed for the corresponding methoxy analogues $\mathbf{5 a}, \mathbf{5 b}$ and $\mathbf{5 c}\left(23,255-24,154 \mathrm{~cm}^{-1}\right)$. On the other hand hydrazone forms of the dyes showed longest emission wavelengths $(21,231-$ $22,831 \mathrm{~cm}^{-1}$ ) among all dyes. Hydrazone forms of dyes show more red shifting emission and lowest Stokes shifts as compared to corresponding azo and methoxy forms of dyes and are in a good agreement with quantum yields. Positive solvatochromism observed for all the dyes presented in (Fig. 4).

\subsubsection{Toluene-PEG 400 mixture}

(a) Spectral data

Absorption spectra of $(\mathbf{4 a}-\mathbf{4 c})$ and $(\mathbf{5 a}-\mathbf{5 c})$ dyes having same concentration were recorded in varying toluene-PEG400 mixture ratio. Dyes (4a-4c) showed dual and broad absorption peaks (azo form and hydrazone form) while only single absorption peak with slight red shift was observed for the corresponding methoxy derivatives (5a-5c) (Fig. S3). Azo forms of dyes (4aA, $\mathbf{4 b A}$ and $\mathbf{4} \mathbf{c A})$ did not exhibit significant emission intensity enhancement in varying ratio of toluene-PEG400 binary mixture (Fig. 5). Calculated relative fluorescence quantum yields and fold of increase at toluene(0.5):PEG400(9.5) v/v compared to toluene for the dyes are 8.6 fold $\left(\Phi_{\mathrm{fl}}=3 \%\right)$ for 4aA, 7.6 fold $\left(\Phi_{\mathrm{fl}}=4 \%\right)$ for $\mathbf{4 b A}$ and 7 fold $\left(\Phi_{\mathrm{fl}}=3 \%\right)$ for 4cA. Similar trends were observed for methoxy analogues, i.e. 5 a showed 7 fold $\left(\Phi_{f l}=2 \%\right)$, 5 b showed 6.5 fold $\left(\Phi_{\mathrm{fl}}=3 \%\right)$ and $\mathbf{5 c}$ showed 7.3 fold $\left(\Phi_{\mathrm{fl}}=4 \%\right)$ at toluene-PEG400 0.5:9.5 (v/v) binary mixture. On the other hand, hydrazone form of dyes $\mathbf{4 a H}, \mathbf{4 b H}$ and $\mathbf{4} \mathbf{c H}$ in toluene-PEG400 binary mixture exhibited significant emission intensity enhancement with increasing PEG 400 content. Hydrazone forms 4aH showed 47.5 fold $\left(\Phi_{\mathrm{fl}}=24 \%\right)$, $\mathbf{4 b H}$ showed 55.6
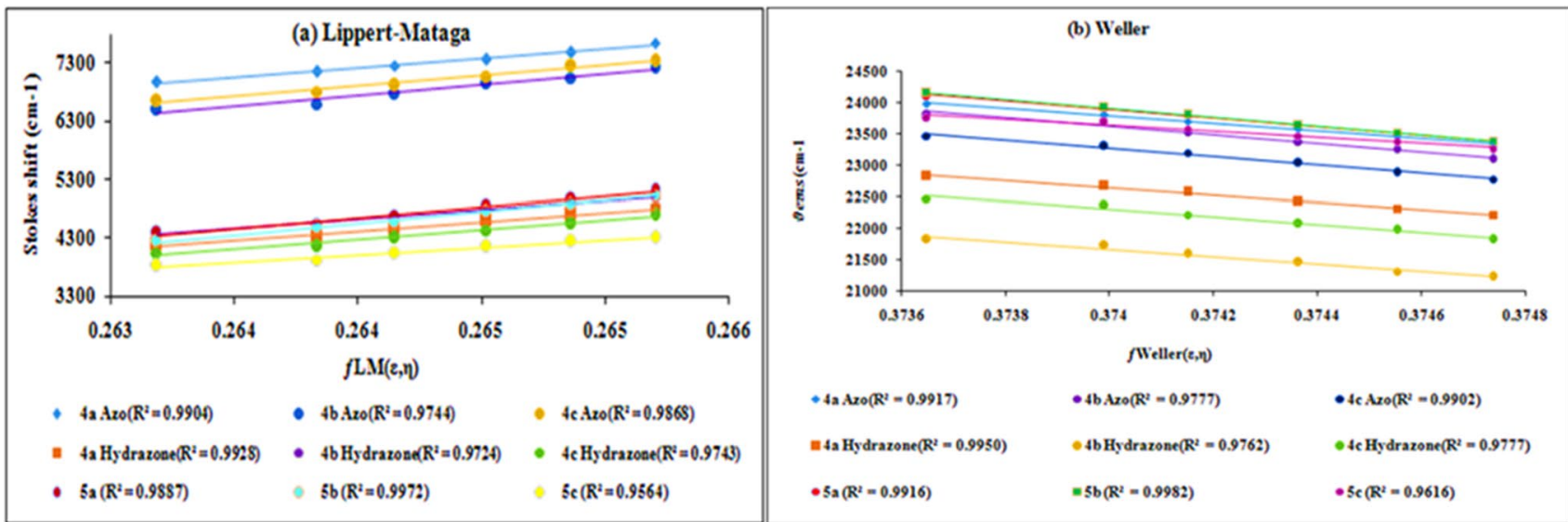

Fig. 4 Weller and Lippert-Mataga plots in different DMSO-Glycerol mixture ratio 

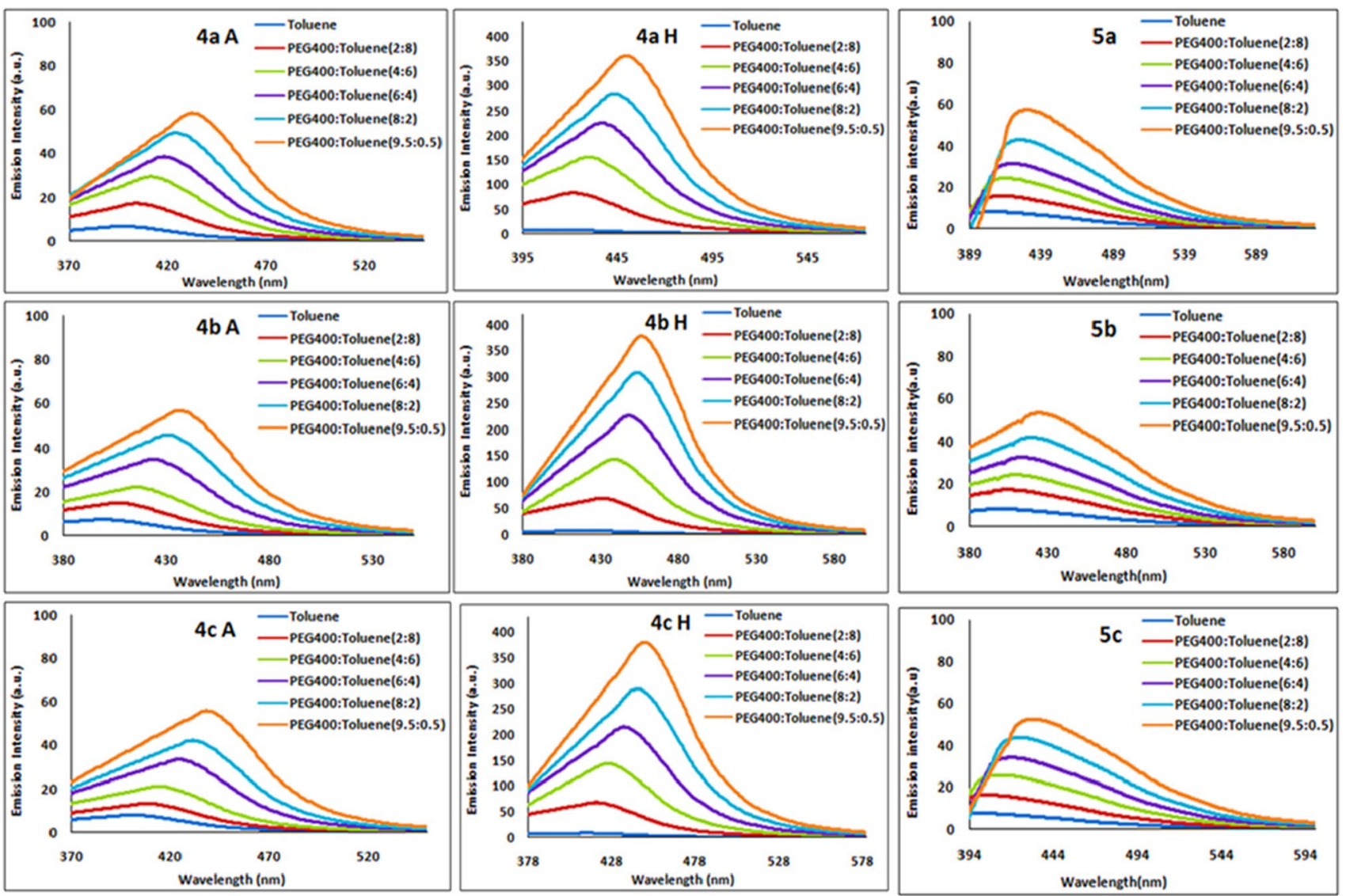

Fig. 5 Emission spectra of $\mathbf{4 a A}$ (excited @ 323-327 nm), 4bA (excited @ 329-333 nm) and 4cA (excited @ 328-332 nm), 5a (excited @ 346-349 nm), 5b (excited @ 361-364 nm) and 5c (excited @ 362-365 nm), 4aH (excited @ 369-373 nm), 4bH (excited @ 380-383 nm) and 4cH (excited @ 377-380 nm) in various toluene-PEG400 mixture ratio fold $\left(\Phi_{\mathrm{fl}}=22 \%\right)$ and $\mathbf{4} \mathbf{c H}$ showed 47.5 fold $\left(\Phi_{\mathrm{fl}}=25 \%\right)$ at toluene-PEG400 (0.5:9.5 v/v) (Fig. 5). In toluenePEG 400 mixture polarity is changing drastically as compared to DMSO-Glycerol mixture, but hydrazone forms of the dyes $(\mathbf{4 a H}, \mathbf{4 b H}$ and $\mathbf{4 c H})$ showed similar emission enhancement in both the viscous medium.

(b) Viscosity sensitivity effect

In toluene-PEG400binary mixtures viscosity increases from $0.55 \mathrm{mPa}$ s (toluene) to $42.21 \mathrm{mPa} \mathrm{s}$ (toluene: PEG400: 5:95). Hydrazone forms of the dyes showed excellent viscosity sensitivity $4 \mathrm{aH}(X=\mathbf{0 . 6 4 6})$, $\mathbf{4 b H}(\mathbf{x}=0.697)$ and $\mathbf{4 c H}(x=0.675)$ with moderately good regression coefficients on excitation @ 372$378 \mathbf{~ n m ~ ( F i g . ~ 6 ) . ~ O n ~ t h e ~ o t h e r ~ h a n d ~ a z o ~ f o r m s ~ 4 a A , ~}$ 4bA and 4CA have exhibited viscosity sensitivity (x) $0.407,0.417$ and 0.413 respectively. Similarly, methoxy analogues $\mathbf{5 a} \mathbf{5} \mathbf{5}$ and $\mathbf{5} \mathbf{c}$ have exhibited viscosity sensitivity $(\mathbf{x}) \mathbf{0 . 3 9 9}, \mathbf{0 . 3 6 6}$ and $\mathbf{0 . 3 8 7}$ respectively. Comparative viscosity trend for all the dyes is found to be $\mathbf{4 b H} \geq \mathbf{4 c H} \geq \mathbf{4 a H}>\mathbf{4 b A} \geq \mathbf{4 c A} \geq \mathbf{4 a A}>5 \mathrm{a} \geq 5 \mathrm{c} \geq$ 5b.

SN Applied Sciences

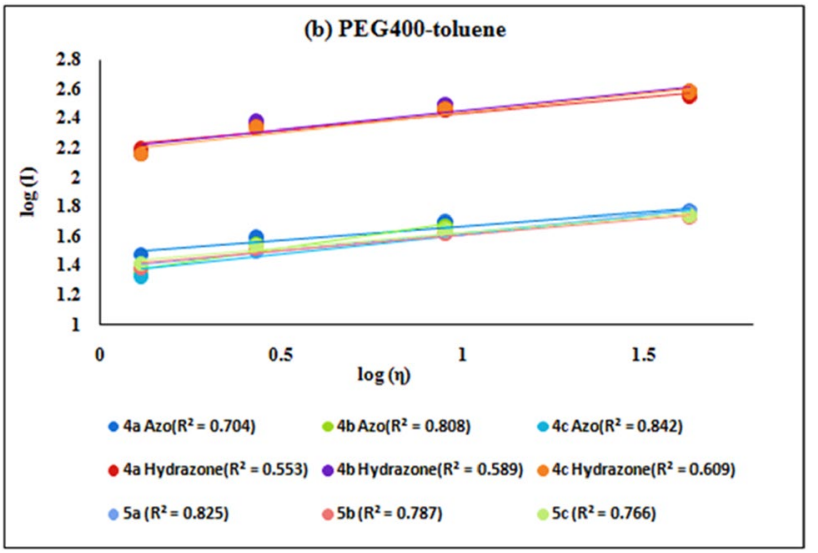

Fig. 6 Comparative viscosity against intensity (logarithmic) spectrum of dyes in differenttoluene-PEG400 mixture ratio

\section{(c) Viscosity polarity effect}

Lippert-Mataga plot showed that azo forms of the dyes $\mathbf{4 a A}, \mathbf{4 b A}$ and $\mathbf{4 c A}$ exhibited positive solvatochromism and large Stokes shifts $5148 \mathrm{~cm}^{-1}$ to 
$7246 \mathrm{~cm}^{-1}$ (Fig. 7). On the other hand small Stokes shifts and positive solvatochromism were observed for corresponding methoxy analogues $\mathbf{5 a}, \mathbf{5 b}$ and $5 c\left(2498-5343 \mathrm{~cm}^{-1}\right)$. Similarly, hydrazone forms of the dyes showed small Stokes shift and positive solvatochromism $\left(2668-4810 \mathrm{~cm}^{-1}\right)$ and are in good correlation to fluorescence quantum yields. Maximum Stokes shifts were observed for $\mathbf{4 a A - 4 c A}$ and found to have very good correlation of having low fluorescence quantum yields. Weller plot showed that azo forms of the dyes (4aA, $\mathbf{4 b A}$ and $\mathbf{4 c A})$ exhibited emis-

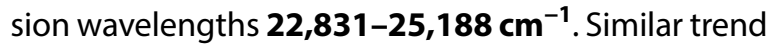
was observed for corresponding methoxy analogues $5 a, 5 b$ and $5 c\left(23,148-25,125 \mathrm{~cm}^{-1}\right)$. On the other hand hydrazone forms of the dyes showed longest

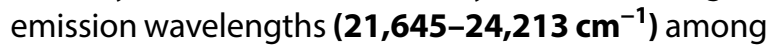
all dyes. Hydrazone forms of dyes exhibited more red shifted emissions and lowest Stokes shifts paralleled to analogous azo and methoxy dyes. Positive solvatochromism has been observed in all the dyes as shown in Fig. 7.

\subsubsection{Toluene-paraffin oil mixture}

\section{(a) Spectral data}

Absorption spectra of dyes in varying toluene-paraffin oil (non-polar viscous medium)were recorded at constant concentration. Dyes $(\mathbf{4 a}-\mathbf{4 c})$ showed dual and broad absorption peaks (azo form and hydrazone form) while only single absorption peak with slight red shift was observed for the corresponding methoxy derivatives (5a-5c) (Fig. S4). Azo forms of dyes (4aA, $\mathbf{4 b A}$ and $\mathbf{4 c A}$ ) did not showed noticeable emission intensity enhancement in varying ratio of toluene-parafin oil binary mixture (Fig. 8). Calculated rela- tive fluorescence quantum yields and fold of increase at toluene(0.5):parafin oil(9.5) $v /$ vin comparison with blank toluene for the azo forms of dyes $4 \mathrm{aA}, 4 \mathbf{b A}$ and 4cA are7.9 fold $\left(\Phi_{f l}=3 \%\right), 6.7$ fold $\left(\Phi_{f l}=5 \%\right)$ and 7.3 fold $\left(\Phi_{\mathrm{fl}}=4 \%\right)$ respectively at toluene-parafin oilo.5:9.5 (v/v) (Fig. 8). Methoxy analogues, i.e. 5a showed 6.5 fold $\left(\Phi_{\mathrm{fl}}=3 \%\right)$, 5 b showed 7.5 fold $\left(\Phi_{\mathrm{fl}}=4 \%\right)$ and 5 c showed 6.6 fold $\left(\Phi_{\mathrm{fl}}=5 \%\right)$ increase at toluene-paraffin oil at 0.5:9.5 (v/v) binary mixture compared to toluene. On the other hand, hydrazone forms $\mathbf{4 a H}$ showed 50.5 fold $\left(\Phi_{\mathrm{fl}}=27 \%\right)$, $\mathbf{4 b H}$ showed 58.9 fold $\left(\Phi_{\mathrm{fl}}=26 \%\right)$ and $\mathbf{4} \mathbf{c H}$ showed 52.1 fold $\left(\Phi_{\mathrm{fl}}=28 \%\right)$ at toluen-paraffin oil 0.5:9.5 (v/v) (Fig. 8). In non-polar viscous mixture also hydrazone forms have found viscosity influenced emission enhancements compared to their respective azo and methoxy forms confirms that hydrogen bonding along with the viscosity played an important role in the emission.

(b) Viscosity sensitivity effect

In toluene-paraffin oil binary mixture viscosity increases from $0.55 \mathrm{mPa}$ s (toluene) to $220.93 \mathrm{mPa} \mathrm{s}$ (toluene:paraffin: 5:95). The hydrazone forms of the dyes show high viscosity sensitivity and are found to be $4 \mathrm{aH}(\mathrm{x}=\mathbf{0 . 4 4 8}), \mathbf{4 b H}(\mathrm{x}=\mathbf{0 . 4 7 9})$ and $4 \mathrm{cH}$ $(\mathbf{x}=\mathbf{0 . 4 5 8})$ with regression coefficients on excitation @ 372-378 nm. On the other hand azo forms 4aA, 4bA and 4cA have exhibited viscosity sensitivity (x) $0.294,0.264$ and 0.269 respectively. Similarly, methoxy analogues $\mathbf{5 a}, \mathbf{5 b}$ and $\mathbf{5 c}$ have exhibited viscosity sensitivity $(x) \mathbf{0 . 2 5 2}, \mathbf{0 . 2 7 0}$ and $\mathbf{0 . 2 5 6}$ respectively. Comparative viscosity trend for all the dyes is found to be in the order $\mathbf{4 b H} \geq \mathbf{4} \mathbf{c H} \geq \mathbf{4 a H}>\mathbf{4 a A} \geq \mathbf{5 b} \geq \mathbf{4} \mathbf{c A}$ $>4 \mathrm{bA}>5 \mathrm{c} \geq 5 \mathrm{a}$ (Fig. 9).

(c) Viscosity polarity effect

Lippert-Mataga plot showed that azo forms of the dyes $\mathbf{4 a A}, \mathbf{4 b A}$ and $\mathbf{4 c A}$ showed positive solvatochromism and large Stokes shifts from $\mathbf{4 8 4 1}$ to
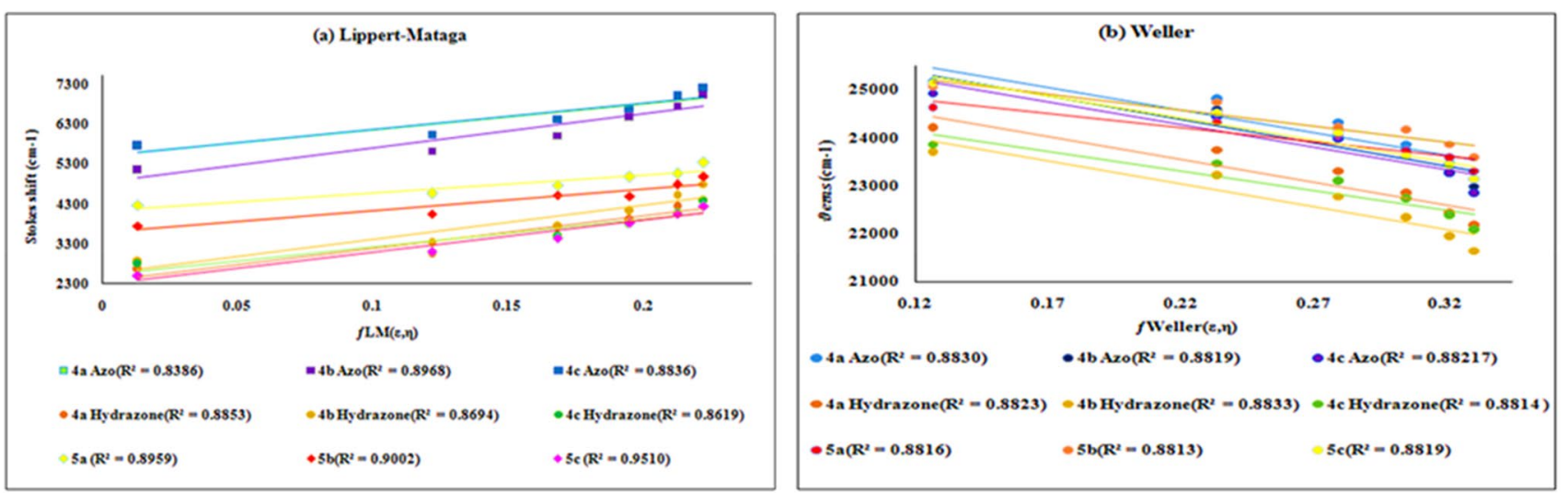

Fig. 7 Lippert-Mataga and Weller plots in toluene-PEG400 mixture ratio 

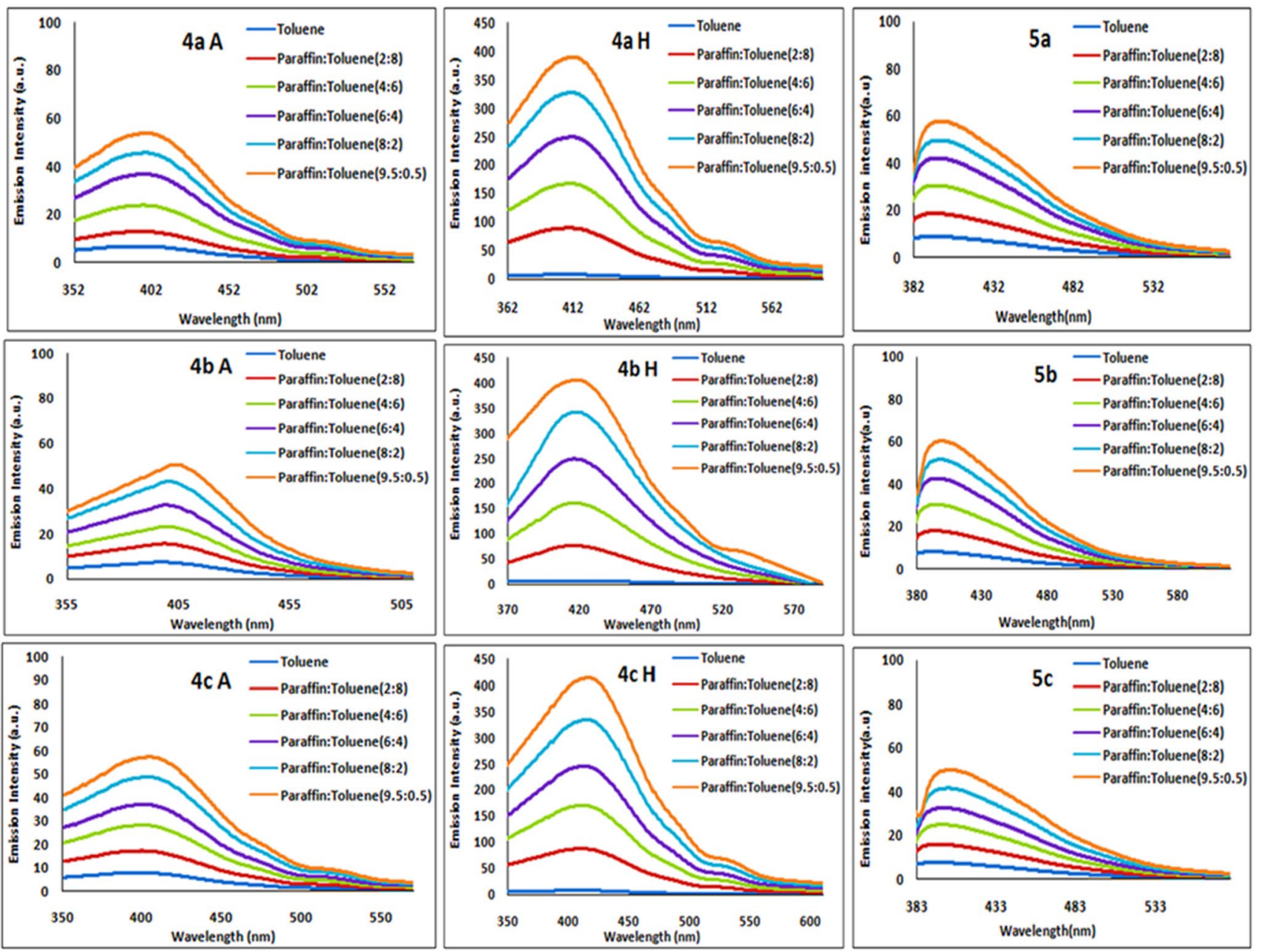

Fig. 8 Emission spectra of 4aA (excited @ 323 nm), 4bA (excited @ 329 nm) and 4cA (excited @ 328 nm), 5a (excited @ 346 nm), 5b (excited @ 361 nm) and 5c (excited @ 362 nm), 4aH (excited @
369 nm), 4bH (excited @ 380 nm) and 4cH (excited @ 377 nm) in various toluene-paraffin oil mixture ratio

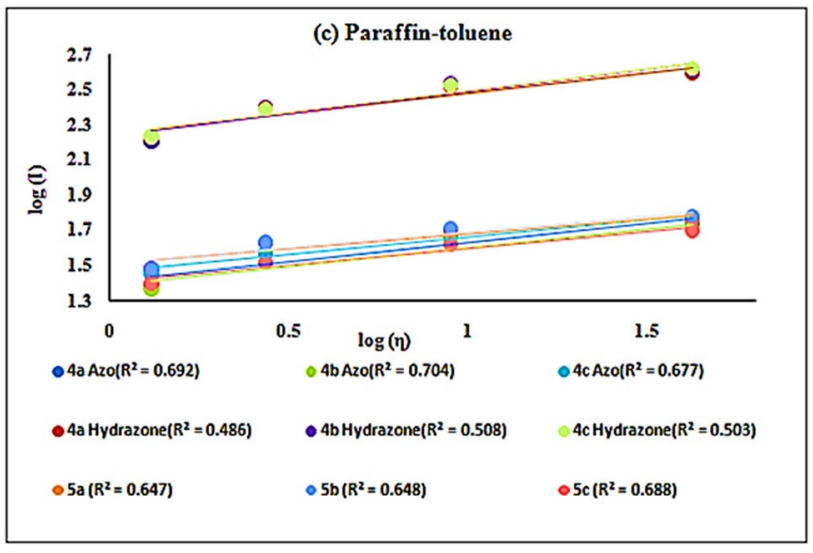

Fig. 9 Comparative viscosity against intensity (logarithmic) spectrum of dyes in different toluene-paraffin oil mixture ratio
$5612 \mathrm{~cm}^{-1}$ (Fig. 10). On the other hand small Stokes shifts and positive solvatochromism were observed for corresponding methoxy analogues $\mathbf{5 a}, \mathbf{5} \mathbf{b}$ and 5c (2307-3755 cm $\left.\mathrm{cm}^{-1}\right)$. Similarly, hydrazone forms of the dyes showed small Stokes shift and positive solvatochromism $\left(2335-2609 \mathrm{~cm}^{-1}\right)$ and is in good correlation to fluorescence quantum yields. Maximum Stokes shifts were observed for 5a-5c. Azo dye and found to have very good correlation of having low fluorescence quantum yields. Weller plot showed that azo forms of the dyes $(\mathbf{4 a A}, \mathbf{4 b A}$ and 4CA) exhibited emission wavelengths in between 24,691 and $25,316 \mathrm{~cm}^{-1}$. Similar trend was observed for corresponding methoxy analogues $\mathbf{5 a}, \mathbf{5} \mathbf{b}$ and $5 c\left(24,813-25,380 \mathrm{~cm}^{-1}\right)$. On the other hand hydrazone forms of the dyes showed emission wavelengths $\left(23,866-24,509 \mathrm{~cm}^{-1}\right)$ among all dyes. Hydrazone forms of dyes showed red shifting emissions and low- 


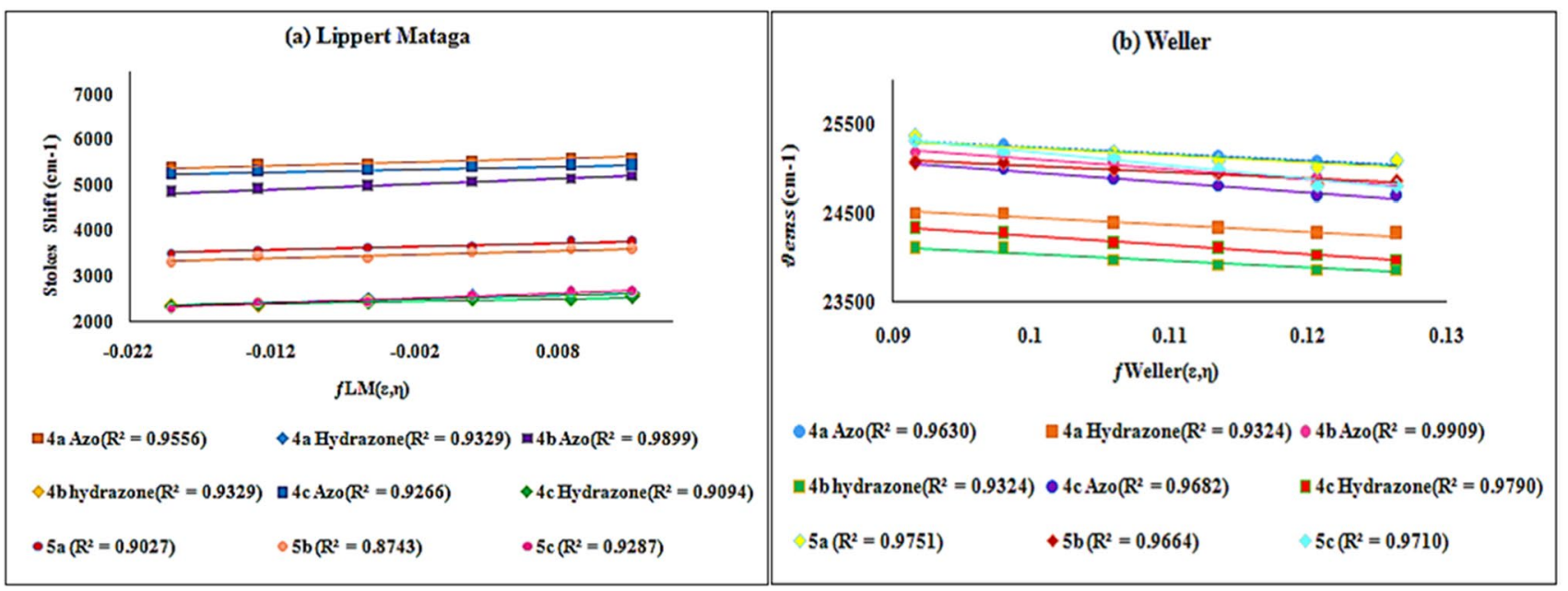

Fig. 10 Lippert-Mataga and Weller plots in toluene-paraffin oil mixture ratio

est Stokes shifts related to analogous azo and methoxy forms and are in good agreement with quantum yields. Positive solvatochromism has been observed for all as shown in Fig. 10.

In summary hydrazone forms of dyes (4aH-4cH) have shown maximum and significant enhancement in emission intensity as compared to azo forms of dyes (4aA-4cA) and parallel methoxy derivatives $(\mathbf{5 a} \mathbf{- 5} \mathbf{c})$ in varying polarity viscous mixtures i.e. DMSO-Glycerol (polar protic), toluene-PEG 400 (mid polar) and toluene-paraffin oil (nonpolar aprotic). This suggested that viscosity and hydrogen bonding (involved in aza-hydrazone tautomerism) are playing crucial role in the enhancement while polarity of the viscous mixture has a significant role in bathochromic shift in the absorption maxima and emission wavelength. We observed good correlation of Stokes shifts $(\Delta \bar{v})$ and fluorescence quantum yields $\left(\Phi_{f}\right)$ of all dyes in the viscous mixture. Hydrazone forms may be attaining more planarity due to hydrogen bonding along with viscosity.

\subsection{Spectral characteristics}

Influence of viscosity and its variable polarity of three viscous binary mixtures on spectral characteristics of dyes has been assessed. It has been identified that with increasing viscous Glycerol, PEG 400 and paraffin oil medium in non-viscous DMSO and toluene medium caused increase in quantum yield $\left(\Phi_{f l}\right)$, radiative rate constant $\left(K_{r}\right)$ and life time $(\tau)$ (Table 2).

All the dyes exhibited positive solvatochromism. Hydrazone forms showed highest fluorescence quantum yields and small Stokes shifts than corresponding azo and methoxy analogues in all the viscous mixtures and are in a good agreement with the radiative rate constant $\left(K_{r}\right)$. Nonradiative rate constant $\left(K_{n r}\right)$ decreases with the increasing viscosity. $K_{n r}>>K_{r}$ clearly indicates that these azo dyes de-excited favorably in non-radiative manner even in the viscous medium (Table 2). Estimated spectral characteristics at the lowest and the highest viscous media of three binary mixtures are presented in Table 2 .

\subsection{Aggregation induced emission enhancement}

Aggregation induced emission enhancement study carried out for synthesized azo dyes with different $\mathrm{H}_{2} \mathrm{O}$ fractions in THF. Dyes (4a-4c) showed two absorptions in the range of $325,331,331 \mathrm{~nm}$ and second absorption at $373,382,375 \mathrm{~nm}$ respectively. Excitation at shorter wavelength did not exhibit any AIEE characteristics for (4a-4c) dyes but, at longer wavelength the dyes exhibited steady change in emission intensity. In THF and $\mathrm{THF} / \mathrm{H}_{2} \mathrm{O}$ mixtures with different $\mathrm{H}_{2} \mathrm{O}$ fractions lower than $50 \%$ dyes exhibited weak fluorescence and fluorescence increased with water fractions from 60 to $90 \%$ in $\mathrm{THF} / \mathrm{H}_{2} \mathrm{O}$ mixture (Fig. 11 and Fig. S2). We noticed that emission intensity also increased at concentration from 20 to $40 \%$ in $\mathrm{THF} / \mathrm{H}_{2} \mathrm{O}$ mixture but compared to the $50 \% \mathrm{THF} / \mathrm{H}_{2} \mathrm{O}$ mixture the emission intensity observed was less. This emission intensity enhancement could be attributed to the restricted rotation of $\mathrm{N}=\mathrm{N}$ bond in solvent-water mixture prominent to formation of molecular aggregates.

\subsection{Color co-ordinates}

$\mathrm{K} / \mathrm{S}$ values of dyes estimated using equation [66]. 
Table 2 Estimated photophysical properties of all dyes in varies viscous mixtures

\begin{tabular}{|c|c|c|c|c|c|c|c|}
\hline Dye & Entry & DMSO & $\begin{array}{l}\text { DMSO:Glycerol } \\
(0.5: 9.5 \mathrm{v} / \mathrm{v})\end{array}$ & Toluene & $\begin{array}{l}\text { Toluene:PEG400 } \\
(0.5: 9.5 \mathrm{v} / \mathrm{v})\end{array}$ & Toluene & $\begin{array}{l}\text { Toluene:paraffinoil } \\
(0.5: 9.5 \mathrm{v} / \mathrm{v})\end{array}$ \\
\hline \multirow[t]{4}{*}{$4 a A$} & $\Phi_{f}$ & 0.44 & 4 & 0.34 & 3 & 0.37 & 3 \\
\hline & $\tau(\mathrm{ns})$ & 0.01 & 0.03 & 0.02 & 0.05 & 0.01 & 0.05 \\
\hline & $K r\left(s^{-1}\right)\left(\times 10^{8}\right)$ & 1.4 & 2.1 & 2.3 & 2.7 & 1.9 & 2.5 \\
\hline & $K_{n r}\left(s^{-1}\right)\left(\times 10^{10}\right)$ & 62.4 & 3.2 & 78.3 & 2.1 & 80 & 1.8 \\
\hline \multirow[t]{4}{*}{$4 \mathrm{aH}$} & $\Phi_{f}$ & 0.31 & 30 & 0.50 & 24 & 0.53 & 27 \\
\hline & $\tau(\mathrm{ns})$ & 0.01 & 0.07 & 0.02 & 0.08 & 0.01 & 0.06 \\
\hline & $K r\left(s^{-1}\right)\left(\times 10^{8}\right)$ & 2.2 & 3.8 & 2 & 3.6 & 1.8 & 2.9 \\
\hline & $K_{n r}\left(s^{-1}\right)\left(\times 10^{10}\right)$ & 73.7 & 0.32 & 80.6 & 0.21 & 59.2 & 0.11 \\
\hline \multirow[t]{4}{*}{$5 a$} & $\Phi_{f}$ & 0.17 & 3 & 0.28 & 2 & 0.46 & 3 \\
\hline & $\tau(\mathrm{ns})$ & 0.01 & 0.06 & 0.02 & 0.04 & 0.01 & 0.03 \\
\hline & $K r\left(s^{-1}\right)\left(\times 10^{8}\right)$ & 7.7 & 10.5 & 3.6 & 5.7 & 6.3 & 8.3 \\
\hline & $K_{n r}\left(\mathrm{~s}^{-1}\right)\left(\times 10^{10}\right)$ & 4.8 & 1.7 & 5.2 & 1.5 & 4.3 & 2.1 \\
\hline \multirow[t]{4}{*}{$4 b A$} & $\Phi_{f}$ & 0.6 & 5 & 0.52 & 4 & 0.74 & 5 \\
\hline & $\tau(\mathrm{ns})$ & 0.02 & 0.05 & 0.01 & 0.04 & 0.02 & 0.06 \\
\hline & $K r\left(s^{-1}\right)\left(\times 10^{8}\right)$ & 3.3 & 5.2 & 2.9 & 4.1 & 2.7 & 3.5 \\
\hline & $K_{n r}\left(s^{-1}\right)\left(\times 10^{10}\right)$ & 8.2 & 1.3 & 7.4 & 2.3 & 5.8 & 1.7 \\
\hline \multirow[t]{4}{*}{$4 \mathrm{bH}$} & $\Phi_{f}$ & 0.34 & 27 & 0.39 & 22 & 0.44 & 26 \\
\hline & $\tau(\mathrm{ns})$ & 0.01 & 0.07 & 0.01 & 0.06 & 0.02 & 0.08 \\
\hline & $K r\left(s^{-1}\right)\left(\times 10^{8}\right)$ & 3.1 & 4.3 & 2.2 & 3.8 & 2.8 & 4.1 \\
\hline & $K_{n r}\left(s^{-1}\right)\left(\times 10^{10}\right)$ & 62.4 & 0.28 & 58 & 0.13 & 71.3 & 0.12 \\
\hline \multirow[t]{4}{*}{$5 \mathbf{b}$} & $\Phi_{f}$ & 0.19 & 3 & 0.46 & 3 & 0.53 & 4 \\
\hline & $\tau(\mathrm{ns})$ & 0.01 & 0.04 & 0.02 & 0.05 & 0.01 & 0.03 \\
\hline & $K r\left(s^{-1}\right)\left(\times 10^{8}\right)$ & 7.2 & 10.2 & 3.5 & 9.2 & 8.3 & 13.2 \\
\hline & $K_{n r}\left(s^{-1}\right)\left(\times 10^{10}\right)$ & 11.4 & 1.8 & 13.5 & 1.2 & 17.8 & 1.1 \\
\hline \multirow[t]{4}{*}{$4 c A$} & $\Phi_{f}$ & 0.5 & 3 & 0.42 & 3 & 0.54 & 4 \\
\hline & $\tau(\mathrm{ns})$ & 0.01 & 0.04 & 0.01 & 0.05 & 0.02 & 0.05 \\
\hline & $K r\left(s^{-1}\right)\left(\times 10^{8}\right)$ & 5.2 & 5.9 & 4.8 & 5.4 & 6.1 & 6.5 \\
\hline & $K_{n r}\left(\mathrm{~s}^{-1}\right)\left(\times 10^{10}\right)$ & 14.2 & 0.8 & 9.8 & 1.3 & 7.3 & 0.9 \\
\hline \multirow[t]{4}{*}{$4 \mathrm{cH}$} & $\Phi_{f}$ & 0.38 & 29 & 0.52 & 25 & 0.53 & 28 \\
\hline & $\tau(\mathrm{ns})$ & 0.02 & 0.06 & 0.02 & 0.08 & 0.01 & 0.07 \\
\hline & $K r\left(\mathrm{~s}^{-1}\right)\left(\times 10^{8}\right)$ & 6.3 & 6.8 & 5.9 & 6.7 & 5.2 & 6.1 \\
\hline & $K_{n r}\left(\mathrm{~s}^{-1}\right)\left(\times 10^{10}\right)$ & 48.3 & 0.3 & 53.7 & 0.43 & 68.4 & 0.3 \\
\hline \multirow[t]{4}{*}{$5 c$} & $\Phi_{f}$ & 0.32 & 4 & 0.54 & 4 & 0.75 & 5 \\
\hline & $\tau(\mathrm{ns})$ & 0.01 & 0.05 & 0.02 & 0.07 & 0.01 & 0.06 \\
\hline & $K r\left(\mathrm{~s}^{-1}\right)\left(\times 10^{8}\right)$ & 7.2 & 7.8 & 6.9 & 7.5 & 6.7 & 7.1 \\
\hline & $K_{n r}\left(s^{-1}\right)\left(\times 10^{10}\right)$ & 15.3 & 2.2 & 17.4 & 1.2 & 11.2 & 1.1 \\
\hline
\end{tabular}

$\mathrm{K} / \mathrm{S}=\frac{(1-R)^{2}}{2 R}$

Where,

$\mathrm{R}=$ reflectance

$\mathrm{K}$ and $\mathrm{S}=$ absorption and scattering coefficients, respectively.

Synthesized disperse azo dyes applied on PET, NYL and ACR fabric at 2\% shade, 1:30 MLR (Material to liquor ratio). The CIELAB system $\left(L^{*}, a^{*}\right.$ and $\left.b^{*}\right)$ used for evaluation of dyed substrate. Dyes on applying to PET dyeing showed that hue (h0) of methylated form is less than compare to hydroxyl form of benzophenone based azo dyes. 4a show highest hue (yellowish) and $\mathbf{4 c}$ show lowest hue (Reddish) (Table 3a). Table 3b showed color coordinates of nylon dyeing where no specific observation observed. All the dyes show hues $\left(\mathrm{h}^{0}\right)$ in between 77.25-89.26 (Yellowish) but K/S values of hydroxyl dyes $(\mathbf{4 a}, \mathbf{4 b}, \mathbf{4 c})$ is slightly greater than methylated dyes $(\mathbf{5 a}$, $\mathbf{5 b}, \mathbf{5 c}$ ). Dyes on applying to acrylic dyeing showed similar observations as that on nylon (Table 3c). 

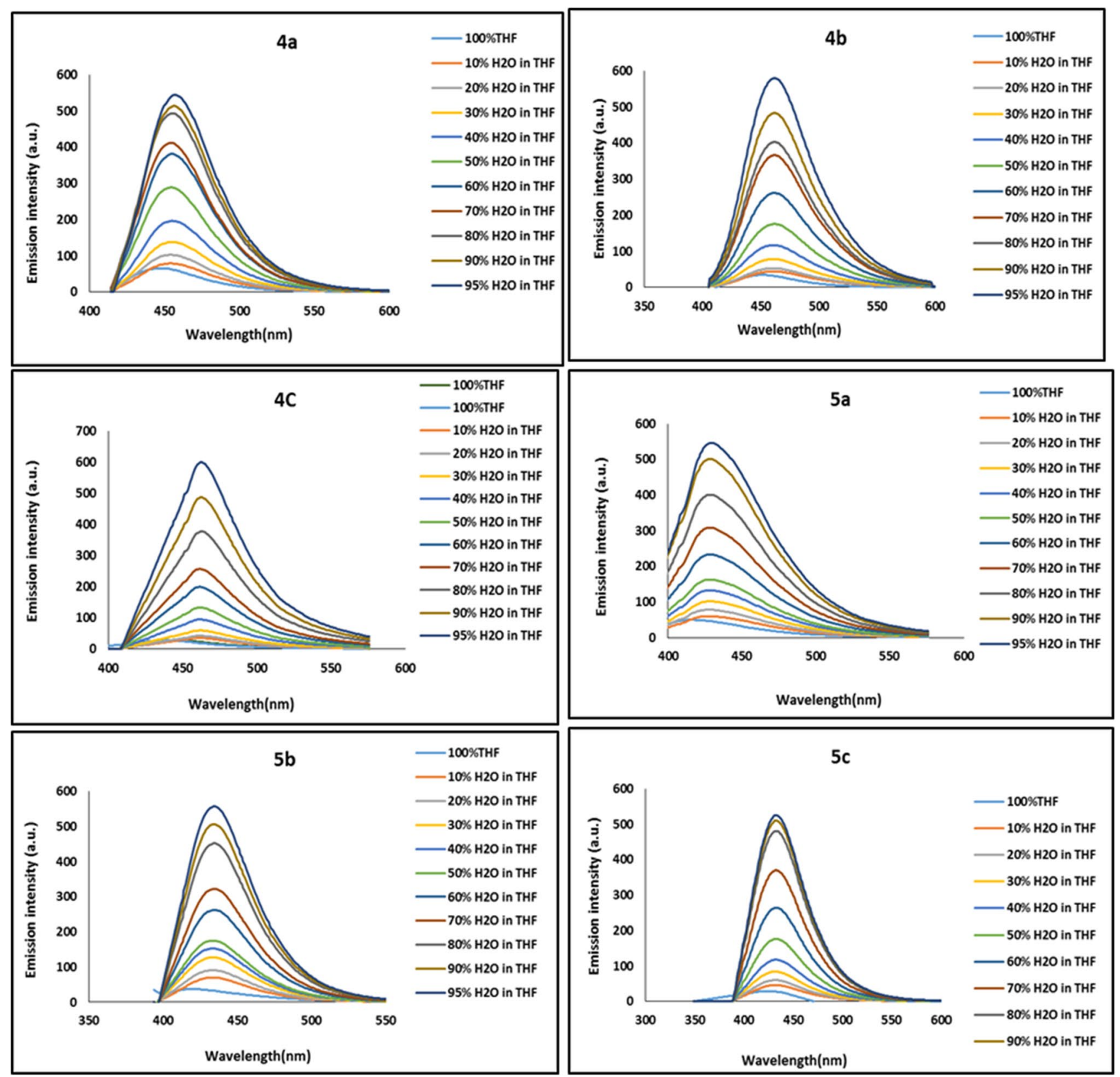

Fig. 11 Emission spectra of $\mathbf{4 a}, \mathbf{4 b}, \mathbf{4} \mathbf{c}$ and $\mathbf{5 a}, \mathbf{5 b}, \mathbf{5} \mathbf{c}$ dyes in $\mathrm{THF}-\mathrm{H}_{2} \mathrm{O}$ mixtures with different water fractions $(5 \mu \mathrm{M})$

Table 3a Color coordinates (CIELAB) for PET dyeing

\begin{tabular}{lllllll}
\hline Dye & $L^{*}$ & $a^{*}$ & $b^{*}$ & $c^{*}$ & $h^{0}$ & $\mathrm{~K} / \mathrm{S}$ \\
\hline 4a & 73.1 & 9.5 & 73.8 & 74.4 & 82.6 & 25.73 \\
4b & 65.5 & 14.4 & 68.6 & 70.1 & 78.1 & 17.28 \\
$\mathbf{4 c}$ & 63.3 & 17.9 & 68.4 & 70.7 & 75.3 & 24.73 \\
$\mathbf{5 a}$ & 50.3 & 21.3 & 57.7 & 61.5 & 69.7 & 2.28 \\
$\mathbf{5 b}$ & 47.7 & 21.7 & 53.8 & 58.1 & 68.0 & 2.26 \\
$\mathbf{5 c}$ & 53.4 & 23.3 & 49.5 & 54.7 & 64.7 & 10.05 \\
\hline
\end{tabular}


On the other hand, dyes bond strongly to PET and are slightly affected by UV radiations $[67,68]$. Hence, PET is more suitable than nylon for the dyes under investigations.

\subsubsection{Fastness properties}

After heat setting at $180^{\circ} \mathrm{C}$ for $60 \mathrm{~s}$ the dyed substrates were tested in a sublimation testing machine at $177^{\circ} \mathrm{C}$ for $30 \mathrm{~s}$. The tested fabrics were rated against the standard Grey scales (1-5 grade) where 1 is poor and 5 is excellent grade for sublimation fastness. Sublimation fastness test result values range from moderate to excellent (Table 4). On comparison between all dyed fabrics PET samples show little better sublimation fastness than acrylic and nylon dyed fabric. The sublimation fastness results in (Table 4) observed that the dyes containing substituent groups such as $-\mathrm{NO}_{2},-\mathrm{Cl}(\mathbf{4} \mathbf{b}, \mathbf{4} \mathbf{c}, \mathbf{5} \mathbf{b}, \mathbf{5} \mathbf{c})$ has better sublimation fastness than unsubstitued (4a, 5a) dyes. Substituted group tend to increase the and hence their sublimation fastness were increased [69]

Comparative light fastness of hydroxyl dyes and corresponding methylated forms of these benzophenone based azo dyes has performed to get insight into role of $\mathrm{H}$-bonding and methylation simultaneously. From Table 4 it is observed that light fastness of all the dyes varies from very good to excellent. On PET fabric dyes show excellent light fastness. On nylon fabric dyes show very good to excellent light fastness. On acrylic fabric dyes show less light fastness rating as compared to PET and nylon.

It is well known that nitro-substituted dyes give poor light fastness on nylon but, in our case dye $\mathbf{4 c}$ and $\mathbf{5 c}$ exhibited very high photostability on nylon. Most commercial disperse dyes are aniline or N,N-dialkylaniline substituted. On such dyes some light fastness—structure
Table 3b Color coordinates (CIELAB) for nylon dyeing

\begin{tabular}{lllllll}
\hline Dye & $L^{*}$ & $a^{*}$ & $b^{*}$ & $c^{*}$ & $h^{0}$ & $\mathrm{~K} / \mathrm{S}$ \\
\hline 4a & 69.4 & 7.9 & 54.4 & 55.0 & 81.7 & 7.89 \\
4b & 68.8 & 0.6 & 47.9 & 47.9 & 89.3 & 3.75 \\
$\mathbf{4 c}$ & 65.1 & 10.5 & 46.4 & 47.5 & 77.3 & 8.06 \\
$\mathbf{5 a}$ & 53.8 & 3.8 & 28.8 & 29.1 & 82.4 & 1.10 \\
$\mathbf{5 b}$ & 59.1 & 6.5 & 35.1 & 35.7 & 79.4 & 1.85 \\
$\mathbf{5 c}$ & 66.4 & 4.4 & 46.5 & 46.7 & 84.5 & 2.75 \\
\hline
\end{tabular}

Table 3c Color coordinates (CIELAB) for acrylic dyeing

\begin{tabular}{lllllll}
\hline Dye & $L^{*}$ & $a^{*}$ & $b^{*}$ & $c^{*}$ & $h^{0}$ & $\mathrm{~K} / \mathrm{S}$ \\
\hline 4a & 57.9 & 13.4 & 48.2 & 50.0 & 74.5 & 14.05 \\
4b & 61.1 & 7.1 & 46.9 & 47.5 & 81.4 & 10.34 \\
4c & 60.8 & 12.5 & 49.9 & 51.5 & 75.9 & 14.52 \\
$\mathbf{5 a}$ & 53.1 & 13.1 & 46.1 & 47.9 & 74.1 & 5.40 \\
$\mathbf{5 b}$ & 44.0 & 7.1 & 31.1 & 31.9 & 77.0 & 2.34 \\
$\mathbf{5 c}$ & 51.3 & 16.0 & 39.3 & 42.4 & 67.8 & 6.85 \\
\hline
\end{tabular}

Table 4 Light and sublimation fastness ratings for dyed PET, nylon and acrylic samples

\begin{tabular}{|c|c|c|c|c|c|c|}
\hline \multirow[t]{4}{*}{ Dye } & \multicolumn{2}{|l|}{ PET } & \multicolumn{2}{|l|}{ Nylon } & \multicolumn{2}{|l|}{ Acrylic } \\
\hline & Light & Sublimation & Light & Sublimation & Light & Sublimation \\
\hline & Fastness rating & Fastness rating & Fastness rating & Fastness rating & Fastness rating & Fastness rating \\
\hline & $(1-8)^{*}$ & $(1-5)^{*}$ & $(1-8)^{*}$ & $(1-5)^{*}$ & $(1-8)^{*}$ & $(1-5)^{*}$ \\
\hline $4 a$ & 8 & $4 / 5$ & $7 / 8$ & 4 & 7 & $3 / 4$ \\
\hline $4 b$ & 8 & 5 & 8 & 5 & $7 / 8$ & 4 \\
\hline $4 c$ & 8 & 5 & 8 & $4 / 5$ & $6 / 7$ & 4 \\
\hline $5 a$ & 8 & $4 / 5$ & $7 / 8$ & 4 & 7 & 3 \\
\hline $5 \mathbf{b}$ & 8 & 5 & $7 / 8$ & $4 / 5$ & 7 & 4 \\
\hline $5 c$ & 8 & 5 & 8 & 5 & 7 & 4 \\
\hline
\end{tabular}

Rating* (Light fastness: 1-poor and 8-Excellent) (Sublimation Fastness: 1-poor and 5-Excellent) 
correlations indicate that the fastness increases as the substituent in the para position is made more electronegative [70] and second substituent in the ortho position have a profound effect on light fastness. Light fastness increases with increasing electron-donating power of the ortho substituents to the azo linkage [71]. The dye $\mathbf{4 c}$ and $\mathbf{5 c}$ is an identical example for this criteria (Scheme 1). Therefore, $p$-nitro dye (4c and $\mathbf{5 c}$ ) being most light fast on nylon.

Comparative light and sublimation fastness of present benzophenone based disperse azo dyes and previously reported disperse azo dyes are tabulated in Table 5. Both hydroxyl and methylated forms of benzophenone based azo dyes show much better sublimation and light fastness as compared to the earlier reported dyes (Table 5).

\subsubsection{Percentage exhaustion determination}

The \% exhaustion of dye bath achieved by collecting sample before and after dyeing. Optical density of each sample were recorded using UV-Vis spectrometer (Perkin Elmer Lambda 25 at $\lambda_{\max }$ ) of the corresponding dye to get concentration of dye ( $\mathrm{g} / \mathrm{l})$. The \% exhaustion (E\%) of dyes on PET, nylon and acrylic fabrics was calculated using equation [72].

$\%$ Exhaustion $=\frac{\text { Initial } \mathrm{OD}_{0}-\text { Final } \mathrm{OD}_{1}}{\text { Iniltial } O D_{0}} \times 100$

Where,

$\mathrm{OD}=$ optical density.

$\mathrm{OD}_{0}=$ optical density before the start of dyeing.

$\mathrm{OD}_{1}=$ optical density at the end of dyeing.

Table 6 represents percentage exhaustion of dyes. All dyes showed exhaustion better on PET, nylon than acrylic substrate.

\subsubsection{Ultraviolet protection factor}

Ultraviolet protection factor (UPF) is the term used to quantify the amount of ultraviolet (UV) protection provided to skin by fabric [73]. Ultra-Protection Factor

Table 5 Comparison of light fastness and sublimation fastness with previous published work

\begin{tabular}{|c|c|c|c|c|c|c|}
\hline \multirow[t]{2}{*}{ Sr. no. } & \multirow[t]{2}{*}{ Structure } & \multicolumn{2}{|l|}{ PET } & \multicolumn{2}{|l|}{ Nylon } & \multirow[t]{2}{*}{ Reference } \\
\hline & & $\begin{array}{l}\text { Light fast- } \\
\text { ness }\end{array}$ & $\begin{array}{l}\text { Sublimation } \\
\text { fastness }\end{array}$ & $\begin{array}{l}\text { Light fast- } \\
\text { ness }\end{array}$ & $\begin{array}{l}\text { Sublimation } \\
\text { fastness }\end{array}$ & \\
\hline 1 & & 6 & 3 & 4 & 3 & [74] \\
\hline 2 & & 7 & 3 & 5 & 4 & [15] \\
\hline 3 & & 8 & 5 & 7 & 4 & This work \\
\hline 4 & & 8 & 5 & 8 & 5 & This work \\
\hline
\end{tabular}


Table 6 Percentage exhaustion and fixation of all dyes on PET, nylon, acrylic

\begin{tabular}{llll}
\hline Dyes no. & $\begin{array}{l}\text { PET (\%) } \\
\text { Exhaustion }\end{array}$ & $\begin{array}{l}\text { Nylon (\%) } \\
\text { Exhaustion }\end{array}$ & $\begin{array}{l}\text { Acrylic (\%) } \\
\text { Exhaustion }\end{array}$ \\
\hline 4a & 83 & 80 & 45 \\
4b & 98 & 87 & 50 \\
4c & 77 & 75 & 42 \\
5a & 91 & 88 & 54 \\
$\mathbf{5 b}$ & 96 & 70 & 44 \\
$\mathbf{5 c}$ & 82 & 72 & 41 \\
\hline
\end{tabular}

(UPF), UV-A blocking [400-320 nm] and UV-B blocking [320-280 nm] were tested to check the UV stability of dyed substrate. UVA range has major effects on skin such as wrinkles, drying, discoloration, skin cornification and photo-toxic reactions with drugs and cosmetics [74].

UPF value measured by calculating mean of percentage transmission in UVA and UVB region using the following equation [75].

$U P F=\frac{\sum_{\lambda=280}^{400} E \lambda S \lambda \Delta \lambda}{\sum_{\lambda=280}^{400} E \lambda S \lambda T \lambda \Delta \lambda}$
Where,

$E \lambda=$ relative erythemal spectral effectiveness

$S \lambda=$ solar spectral irradiance

$\mathrm{T} \lambda=$ average spectral transmission of the specimen

$\Delta \lambda=$ measured wavelength interval $(\mathrm{nm})$

Table 7 shows UPF ratings and UVA and UVB blocking values of dyed substrate. A molecules contains benzophenone as core moiety and it known for its photo stabilizer property.

\subsection{DFT study}

In order to evaluate and correlated experimental observations with theoretical calculations DFT computations were performed. We have evaluated azo-hydrazone tautomerization [76], stable tautomeric forms and correlated photophysical interpretations with the DFT calculations.

\subsubsection{Geometry optimization}

To know the insight details of the azo and hydrazone forms geometries, computational calculations were performed by using DFT (B3LYP /6-31G(d)). Azo and

Table 7 UPF rating and UVA/UVB blocking in comparison with blank dyed fabric

\begin{tabular}{|c|c|c|c|c|c|c|c|c|c|}
\hline \multirow[t]{2}{*}{ Dye } & \multicolumn{3}{|l|}{ PET } & \multicolumn{3}{|l|}{ Nylon } & \multicolumn{3}{|l|}{ Acrylic } \\
\hline & UPF rating & $\begin{array}{l}\text { UVA Blocking } \\
\text { (\%) }\end{array}$ & $\begin{array}{l}\text { UVB Blocking } \\
\text { (\%) }\end{array}$ & $\begin{array}{l}\text { UPF rating } \\
\text { (\%) }\end{array}$ & $\begin{array}{l}\text { UVA Blocking } \\
\text { (\%) }\end{array}$ & $\begin{array}{l}\text { UVB Blocking } \\
\text { (\%) }\end{array}$ & $\begin{array}{l}\text { UPF rating } \\
\text { (\%) }\end{array}$ & $\begin{array}{l}\text { UVA Blocking } \\
\text { (\%) }\end{array}$ & $\begin{array}{l}\text { UVB Blocking } \\
\text { (\%) }\end{array}$ \\
\hline Blank & 17 & 95.58 & 88.01 & 15 & 66.87 & 67.58 & 20 & 91.58 & 95.90 \\
\hline $4 a$ & 22 & 95.16 & 95.38 & 17 & 74.81 & 74.74 & 50 & 97.56 & 97.94 \\
\hline $4 b$ & 21 & 94.96 & 95.08 & 15 & 65.87 & 66.14 & 37 & 96.78 & 97.47 \\
\hline $4 c$ & 23 & 94.97 & 95.33 & 17 & 63.10 & 63.27 & 33 & 96.25 & 97.17 \\
\hline $5 a$ & 17 & 92.89 & 94.33 & 16 & 65.07 & 65.23 & 37 & 96.40 & 97.35 \\
\hline $5 b$ & 17 & 93.02 & 94.07 & 15 & 66.60 & 67.18 & 25 & 94.37 & 96.36 \\
\hline $5 c$ & 18 & 93.64 & 94.44 & 16 & 65.68 & 66.26 & 30 & 95.17 & 96.57 \\
\hline
\end{tabular}

Rating* (15-24 good protection, $25-39$ very good protection, $40-50,50+$ excellent protection)
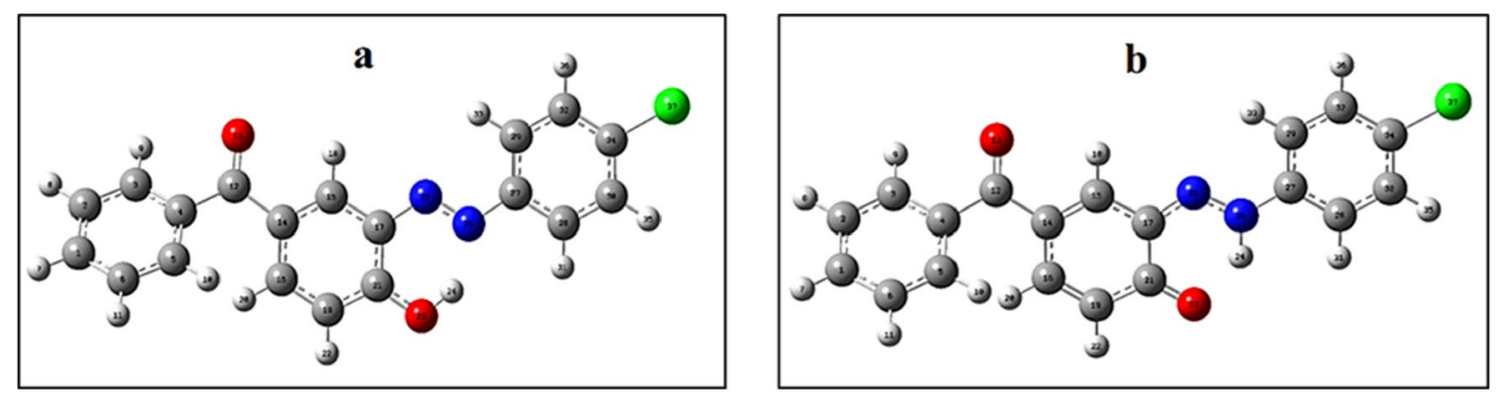

Fig. 12 Optimized geometry of $\mathbf{4 b}$ (a) Azo isomer (b) Hydrazone isomer using B3LYP/6-31G(d) level 
Table 8 Bond lengths of between selected atoms of tautomeric forms (4aA-4cA and $\mathbf{4 a H}-\mathbf{4 c H})$

\begin{tabular}{llllll}
\hline $\begin{array}{l}\text { Com- } \\
\text { pound } \\
\text { name }\end{array}$ & $\begin{array}{l}\mathrm{N}=\mathrm{N} \\
\text { bond } \\
\text { length } \\
(\AA)\end{array}$ & $\begin{array}{l}\mathrm{O}-\mathrm{H} \\
\text { bond } \\
\text { length } \\
(\AA)\end{array}$ & $\begin{array}{l}\mathrm{N}-\mathrm{H} \\
\text { bond } \\
\text { length }(\AA)\end{array}$ & $\begin{array}{l}\mathrm{N} \text { atom } \\
\text { no }\end{array}$ & $\mathrm{H}$ atom no \\
\hline $\mathbf{4 a A}$ & 1.2695 & 1.00099 & 1.71397 & 26 & 24 \\
$\mathbf{4 a H}$ & 1.29317 & 1.70561 & 1.04186 & 26 & 24 \\
$\mathbf{4 b A}$ & 1.27002 & 0.99981 & 1.72011 & 26 & 24 \\
$\mathbf{4 b H}$ & 1.2952 & 1.70376 & 1.04188 & 26 & 24 \\
$\mathbf{4} \mathbf{c A}$ & 1.2715 & 0.99926 & 1.72326 & 26 & 24 \\
$\mathbf{4 c H}$ & 1.30214 & 1.72029 & 1.03922 & 26 & 24 \\
\hline
\end{tabular}

Table 9 Calculated energies of tautomeric forms at B3LYP/6-31G(d) in $\mathrm{CHCl}_{3}$

\begin{tabular}{llll}
\hline $\begin{array}{l}\text { Compound } \\
\text { name }\end{array}$ & $\mathrm{E} /$ Hartree & $\Delta \mathrm{E} / \mathrm{kJ} \mathrm{mol}^{-1}$ & $\Delta \mathrm{G} /$ Hartree \\
\hline $\mathbf{4 a A}$ & -992.078 & 0 & -992.144639 \\
$\mathbf{4 a H}$ & -992.0761 & 4.776694 & -992.14282 \\
$\mathbf{4 b A}$ & -1451.682 & 0 & -1451.751916 \\
$\mathbf{4 b H}$ & -1451.6794 & 7.06394 & -1451.749226 \\
$\mathbf{4 c A}$ & -1196.5751 & 0 & -1196.648555 \\
$\mathbf{4 c H}$ & -1196.5737 & 4.603378 & -1196.646802
\end{tabular}

hydrazone forms optimized geometries at Ground state indicated alteration in bond lengths. Optimized geometries of dye $\mathbf{4} \mathbf{b}$ azo-hydrazone forms at ground state in chloroform confirms tautomerization in the dye (Fig. 12).

Further evaluation of aza-hydrazone tautomerism based on bond distances between the selected atoms of the dyes were done (Table 8 ). $-\mathbf{N}=\mathbf{N}$ - bond distances have been increased from 1.2695-1.2715 $\AA$ in azo form to 1.29317-1.30214 $\AA$ in hydrazone form. $-\mathrm{OH}$ bond lengths also increased from 0.99926-1.00099 $\AA$ in azo forms to 1.70376-1.72029 Å in hydrazone form. Similarly, -NH bond length get reduced from 1.71397-1.72326 A of azo forms to 1.03922-1.04188 $\AA$ of hydrazone form suggesting clearly the existence of two forms.

\subsubsection{Electronic energies of tautomeric forms}

Two divergent peaks of absorption spectra for these dyes indicate two tautomeric forms, so one can study comparative stabilities of the tautomer forms. Electronic energies of optimized azo forms and respective hydrazone forms in chloroform have been matched using B3LYP functional 6-31G(d) basis set. It is clearly noticeable that all the azo forms of dyes are comparatively more stable than their respective hydrazone forms by 4.60-7.06 kJ mol${ }^{-1}$ (Table 9).

\subsection{TD-DFT computations}

The optimized ground state geometries of the dyes in various polarities solvents were exposed to TD-DFT with B3LYP functional and 6-31G (d) basis set level. As minimum as ten excited states considered for each dye. Absorption maxima, oscillator strengths, vertical excitations, and corresponding orbital contributions in chloroform were recorded (Table 10). DFT and TD-DFT outcomes advise that there is noticeable impact of donating moieties on the absorption of the dyes. Computationally obtained values for the dyes are in decent agreement with experimental interpretations.

Table 10 Observed UV-visible absorption and computed absorption spectral data at B3LYP/6-31G (d) for all dyes in chloroform

\begin{tabular}{|c|c|c|c|c|c|c|c|}
\hline \multicolumn{4}{|c|}{ Experimental } & \multicolumn{4}{|l|}{ Theoretical } \\
\hline Compound & $\lambda_{\max }(\mathrm{nm})$ & $\begin{array}{l}\varepsilon \text { Molar absorptivity } \\
\left(\mathrm{dm}^{3} \mathrm{~mol}^{-1} \mathrm{~cm}^{-1}\right)\end{array}$ & $\begin{array}{l}\text { Oscillator } \\
\text { strength }(\mathrm{f})\end{array}$ & $\begin{array}{l}\text { Vertical excita- } \\
\text { tion }(\mathrm{nm})\end{array}$ & Energy $(\mathrm{eV})$ & $\begin{array}{l}\text { Oscillator } \\
\text { strength (f) }\end{array}$ & $\begin{array}{l}\text { Orbital } \\
\text { contribu- } \\
\text { tion (\%) }\end{array}$ \\
\hline $4 a A$ & 322 & 33,511 & 0.3588 & 326 & 3.8019 & 0.3498 & $\mathrm{H}->\mathrm{L}(95)$ \\
\hline $4 \mathrm{aH}$ & 372 & 18,619 & 0.1713 & 360 & 3.4447 & 0.1896 & $\mathrm{H}->\mathrm{L}(91)$ \\
\hline $4 \mathrm{bA}$ & 332 & 27,671 & 0.8302 & 352 & 3.5195 & 0.8267 & $\mathrm{H}->\mathrm{L}(98)$ \\
\hline $4 \mathrm{bH}$ & 378 & 17,040 & 0.1417 & 399 & 3.1082 & 0.1327 & $\mathrm{H}->\mathrm{L}$ (89) \\
\hline 4CA & 326 & 15,296 & 0.1579 & 349 & 3.5522 & 0.1485 & $H->L(97)$ \\
\hline $4 \mathrm{cH}$ & 378 & 5079 & 0.0409 & 374 & 3.3183 & 0.0457 & $H->L(90)$ \\
\hline $5 \mathrm{aA}$ & 351 & 13,944 & 0.2563 & 359 & 3.4557 & 0.2398 & $H->L(96)$ \\
\hline $5 \mathrm{bA}$ & 360 & 8993 & 0.1564 & 368 & 3.3677 & 0.1731 & $H->L(95)$ \\
\hline $5 C A$ & 376 & 3697 & 0.0633 & 362 & 3.4226 & 0.0313 & $\mathrm{H}->\mathrm{L}$ (99) \\
\hline
\end{tabular}

$\lambda \max =$ Experimental maximum wavelength, $f=$ Oscillator strength 


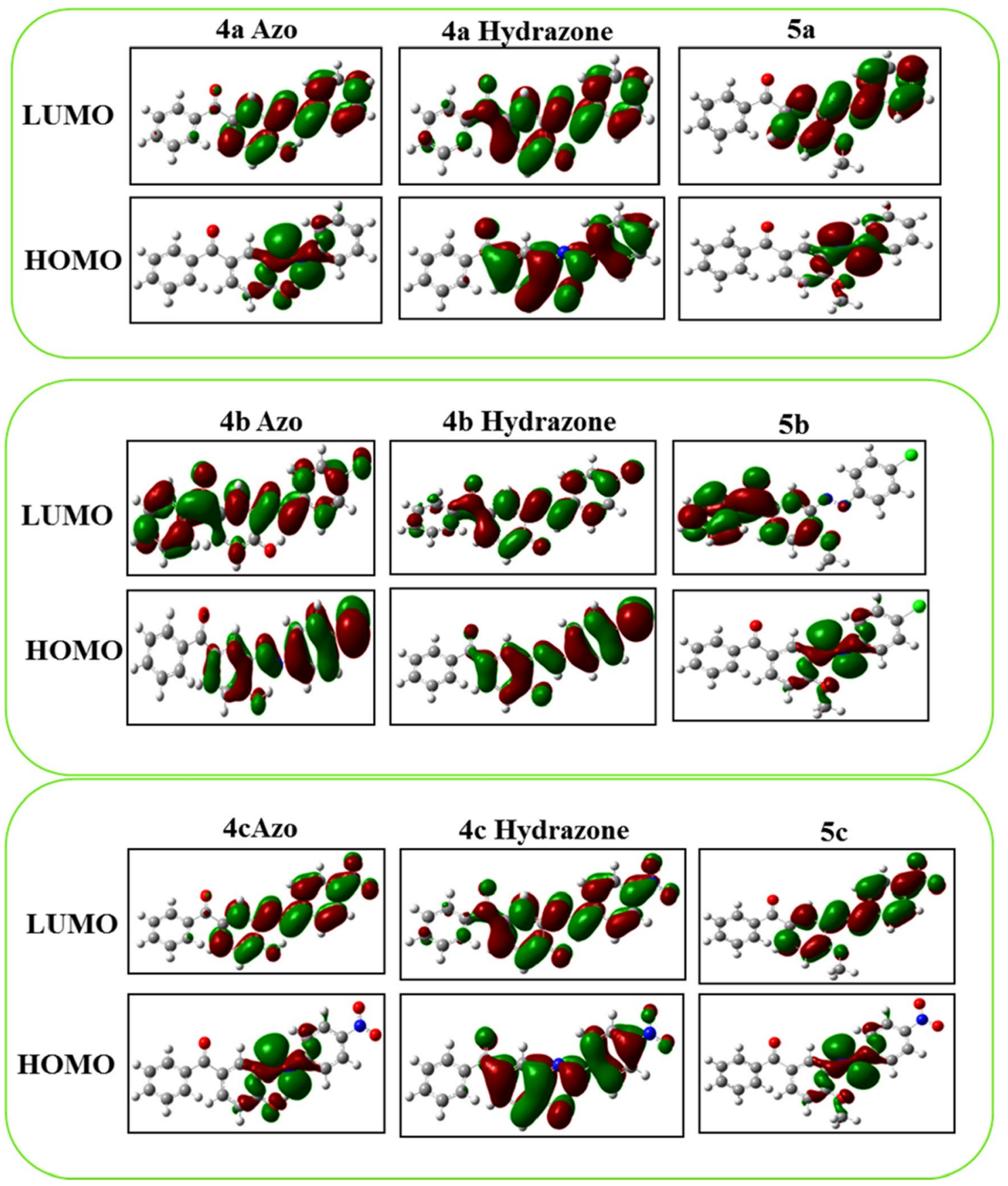

Fig. 13 Frontier molecular orbitals diagrams in $\mathrm{CHCl}_{3}$ optimized at $\mathrm{B} 3 \mathrm{LYP} / 6-31 \mathrm{G}(\mathrm{d})$ level. Optimized structure of $4 \mathrm{~b}$ at $\mathrm{B} 3 \mathrm{LYP} / 6-31 \mathrm{G}(\mathrm{d})$ level (a) Azo form (b) Hydrazone form

\subsubsection{Frontier Molecular orbitals}

Electronic density distributions at highest occupied molecular orbital level (HOMO) and at lowest unoccupied molecular orbital level (LUMO) were assessed through Frontier Molecular Orbitals during excitations. Electron density distribution for azo forms (4aA, $\mathbf{4 b \mathbf { b }}$ and $4 \mathrm{CA}$ ) and corresponding methoxy analogues (5a, $\mathbf{5 b}$ and $\mathbf{5 c}$ ) are situated mostly on $-\mathrm{N}=\mathrm{N}$ - core which is slightly shifted to hydroxyaryl core resulting into less charge transfer and are in good agreement with the spectral data (Fig. 13).

On the other hand, electronic density at HOMO of hydrazone forms $(\mathbf{4 a H}, \mathbf{4} \mathbf{b H}$ and $\mathbf{4} \mathbf{c H})$ is situated mostly on $-\mathrm{N}=\mathrm{N}$ - core and adjacent phenyl moieties which get transferred to the carbonyl of benzophenone 
Fig. 14 HOMO-LUMO energy gap in $\mathrm{CHCl}_{3}$ optimized at B3LYP/6-31G(d) level

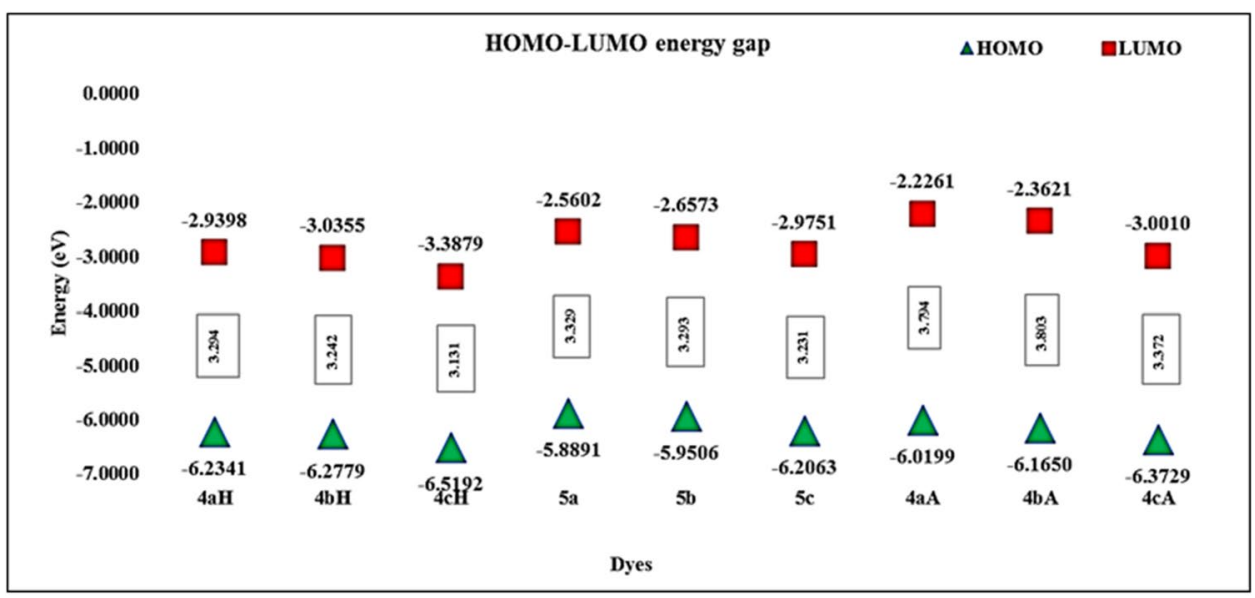

core resulting into more red shift in the photophysical properties.

\subsubsection{HOMO-LUMO energy gap}

HOMO and LUMO energy band gaps of dyes $\mathbf{4 a H}, \mathbf{4} \mathbf{b H}$ and $\mathbf{4} \mathrm{cH}$ hydrazone are lowered than the corresponding optimized azo forms (4aA, 4bA and 4cA) and methoxy forms (5a, 5b and 5c) supported red shifted absorption maxima. Moreover, energy separation between HOMO and LUMO levels follow the same trends suggested large Stokes shift in the hydrazone-form of dyes in comparison to their corresponding azo analogues.- $\mathrm{NO}_{2}$ substituted dyes (4cA, $\mathbf{4} \mathbf{c H}$ and $\mathbf{5 c}$ ) are having more red shift than $-\mathrm{Cl}$ substituted (4bA, $\mathbf{4 b H}$ and $\mathbf{5 b}$ ) dyes than unsubstitued (4aA, 4aH and 5a) dyes (Fig. 14).

\subsection{Electrophilicity index}

Stability of dyes were estimated mathematically by means of computationally gathered electronic energies at B3LYP/6-31G (d) level. So, $\omega$ (electrophilicity index) was utilized. Parr et al. defined the electrophilicity-index as the measurement of tendency of the group to absorb electrons and is mathematically expressed as [77, 78]:

$\omega=\frac{\mu^{2}}{2 \eta}$

Where,

$\omega=$ electrophilicity-index

$\mu=$ chemical-potential

$\eta=$ chemical-hardness

Chemical-hardness and chemical-potential determined by using the following equations:
$\mu=-\frac{(I P+E A)}{2}$

$\eta=\frac{(I P-E A)}{2}$

Where,

$\mathrm{IP}=$ ionization potential, (energy change to the system when an electron is get removed)

$E A=$ electron affinity, (energy change to the system when an electron get added)

But then for finding $E A$ and IP, one can determine three energy values $\left(E_{N}, E_{N-1}\right.$ and $\left.E_{N+1}\right)$

Where,

E symbolises for total energy of a system and $\mathrm{N}$ symbolises total electron counts.

Above said methods make the whole system time consuming and monotonous. So alternatively values of chemical hardness and chemical potential can be determined by using Koopmans theorem:

$\mu=\frac{\left(E_{\text {LUMO }}+E_{\text {HOMO }}\right)}{2}$

$\eta=\frac{\left(E_{\text {LUMO }}-E_{\text {HOMO }}\right)}{2}$

Where,

$\mathrm{E}_{\mathrm{LUMO}}=$ energy associated with LUMO

$\mathrm{E}_{\mathrm{HOMO}}=$ energy associated with $\mathrm{HOMO}$

Also, the Net electrophilicity is expressed as,

$\omega^{ \pm}=\omega^{+}+\omega^{-}$

Where, 


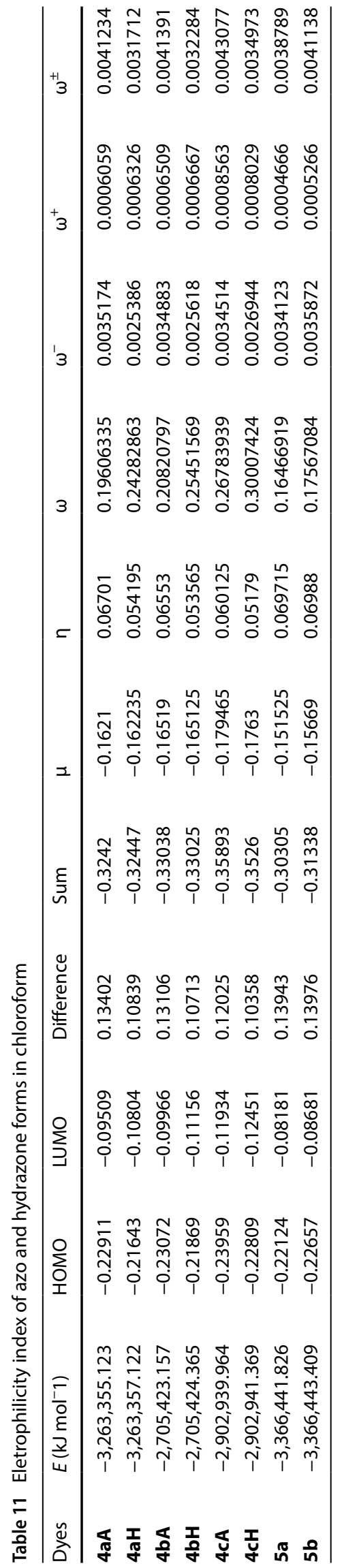

$\omega^{+}=\frac{\left(E_{\text {LUMO }}\right)^{2}}{2\left(E_{\text {LUMO }}-E_{\text {HOMO }}\right)}$

and

$\omega^{-}=\frac{\left(E_{\text {HOMO }}\right)^{2}}{2\left(E_{\text {LUMO }}-E_{\text {HOMO }}\right)}$

Hence, among possible tautomeric structure's, stable confirmation can be predicted by calculating net elerophilicity index $\left(\omega^{ \pm}\right)($Table 11).

\section{Conclusion}

Dyes $\mathbf{4 a} \mathbf{a} \mathbf{4 b}$ and $\mathbf{4 c}$ exhibited dual absorption peaks (azo form and hydrazone forms) and exhibited elivated molarextinction coefficients as related to corresponding methoxy analogues 5a-chaving singal absorption. Azo forms (4aA, $\mathbf{4 b A}$ and $\mathbf{4 c A})$ and their methoxy analogues (5a-c) are less sensitive to the viscosity in varying polarity viscous media (glycerol-dimethylsulfoxide, toluene-polyethylene glycol 400 and toluene-paraffin oil). However, hydrazone forms (4aH, $\mathbf{4 b H}$ and $\mathbf{4} \mathbf{c H}$ ) exhibited highly viscosity sensitive emission enhancement of (47-96 fold) in varying polarities viscous mixtures as a combined effect of viscosity and $\mathrm{H}$-bonding. Systematic substitutional variations along with comprehensive photophysical characterization of 4-hydroxy benzophenone and corresponding parallel methoxy analogues (4-methoxy benzophenone) dyes allowed to evaluate the role of $\mathrm{H}$-bonding in the emission enhancement. Forster-Hoffman plots showed viscosity sensitivity ( $x=0.448-0.697$ ) for hydrazone forms of the dyes $(\mathbf{4} \mathbf{a H}, \mathbf{4} \mathbf{b H}$ and $\mathbf{4 c H})$. Solvent polarity graphs showed positive solvatochromism for all the dyes. Theoretical calculations by DFT revealed azo-hydrazone tautomerization in 4-hydroxy benzophenone series of dyes. Electrophilicity index calculations and HOMO-LUMO energy levels suggested that hydrazone forms are most stable. TD-DFT, Frontier molecular orbitals and HOMO-LUMO energy gap calculations are in good agreement with the experimentally noticed data.

Acknowledgments Suvidha S. Shinde is thankful to UGC-SAP for economic support. Amol G. Jadhav is thankful to UGC for economic support by way of SRF.

\section{Compliance with ethical standards}

Conflict of Interest The author(s) declare that they have no competing interests. 


\section{References}

1. Hunger K (2003. ISBN 3-527-30426-6; Journal of the American Chemical Society 2003;125:10144-10144) Industrial dyes: chemistry, properties, applications Edited by Klaus Hunger (Kelkheim, Germany). Wiley-VCH, Weinheim. https://doi.org/10.1021/ja033 5418

2. Sancho MI, Jubert AH, Blanco SE, Ferretti FH, Castro EA (2008) Determination of dissociation constants of p-hydroxybenzophenone in aqueous organic mixtures-solvent effects. Can J Chem 86:462-469. https://doi.org/10.1139/v08-040

3. Patel HM (2014) Synthesis, structure investigation and dyeing assessment of novel bisazo disperse dyes derived from UV absorbing material. IOSR J Appl Chem 6:51-55

4. Jiang X, Rui Y, Chen G (2009) Improved properties of cotton by atmospheric pressure plasma polymerization deposition of sericin. J Vinyl Addit Technol 21:129-133. https://doi. org/10.1002/vnl

5. Patel HM, Dixit BC (2014) Synthesis, characterization and dyeing assessment of novel acid azo dyes and mordent acid azo dyes based on 2-hydroxy-4-methoxybenzophenone-5-sulfonic acid on wool and silk fabrics. J Saudi Chem Soc 18:507-512. https:// doi.org/10.1016/j.jscs.2011.10.015

6. Dixit B, Patel H, Desai D (2007) Synthesis and application of new mordent and disperse azo dyes based on 2,4-dihydroxybenzophenone. J Serb Chem Soc 72:1 19-127. https://doi.org/10.2298/ JSC0702119D

7. Youssef BM, Ahmed MHM, Arief MMH, Mashaly HM, Abdelghaffar RA, Mahmoud SA (2014) Synthesis and application of functional (Anti-UV) azo-dyes based on $\gamma$-acid on wool fabrics. Indian J Sci Technol 7:1005-1013

8. Kiguchi M, Evans P, Ekstedt J, Williams R, Kataoka Y (2001) Improvement of the durability of clear coatings by grafting of UV-absorbers on to wood. Surface Coat Int Part B: Coat Trans 84:263-270. https://doi.org/10.1007/BF02700407

9. Allen NS (2008) Photofading mechanisms of dyes in solution and polymer media. Rev Progr Color Re Top 17:61-71. https:// doi.org/10.1111/j.1478-4408.1987.tb03752.x

10. Kehayoglou $A H$, Tsatsaroni EG, Kehayoglou AH, Eleftheriadis IC, Kyriazis LE (1998) Effectiveness of various UV-absorbers on the dyeing of polyester with disperse dyes. Part IV. Dyes Pigments 38:65-75. https://doi.org/10.1016/S0143-7208(97)00085-5

11. Kehayoglou AH, Tsatsaroni EG, Eleftheriadis IC, Loufakis KC, Kyriazis LE (1997) Effectiveness of various UV-absorbers in dyeing of polyester with disperse dyes. Part III. Dyes Pigments 34:207-218

12. Tsatsaroni EG, Kehayoglou AH (1995) Dyeing of polyester with $\mathrm{Cl}$ disperse yellow 42 in the presence of various UV-absorbers. Part II. Dyes Pigments 28:123-130. https://doi.org/10.1016/01437208(95)00007-3

13. Tsatsaroni EG, Eleftheriadis IC (2004) UV-absorbers in the dyeing of polyester with disperse dyes. Dyes Pigments 61:141-147. https://doi.org/10.1016/j.dyepig.2003.10.002

14. Freeman HS, Posey JC (1992) An approach to the design of lightfast disperse dyes-analogs of disperse yellow 42. Dyes Pigments 20:171-195

15. Bhide R, Jadhav AG, Sekar N (2016) Light fast monoazo dyes with an inbuilt photostabilizing unit: synthesis and computational studies. Fibers Polym 17:349-357. https://doi.org/10.1007/s1222 1-016-5717-3

16. Dixit BC, Patel HM, Desai DJ, Dixit RB (2009) Studies on dyeing performance of novel acid azo dyes and mordent acid azo dyes based on 2,4-dihydroxybenzophenone. E-J Chem 6:315-322

17. Athalye A (2015) Automotive textiles. Int J Textile Eng Processes $1: 42-52$

18. Zimniewska M. Innovations in UV protection by textiles 2015:35.
19. Farzana N (2015) Fluorescent disperse dyes reflectance spectra, relative strength and performance evaluation of coumarin class on polyester/cotton blended fabric. Int J Res Eng Technol 4:488-494

20. Maqbool H, Ganesh SD, Pai VK (2013) Studies on physicochemical properties of azo cardanol sulfonates and their salts. Der Pharma Chem 5:154-159

21. Szuster L, Kaźmierska M, Król I (2004) Fluorescent dyes destined for dyeing high-visibility polyester textile products. Fibres Textiles Eastern Europe 12:70-75

22. Yang P, Kokot $S$ (1996) Thermal analysis of different cellulosic fabrics. J Appl Polym Sci 60:1137-1146. https://doi.org/10.1002/(SICI)1097-4628(19960 523)60:8<1137::AID-APP6>3.0.CO;2-M

23. Barsotti F, Brigante M, Sarakha M, Maurino V, Minero C, Vione D (2015) Photochemical processes induced by the irradiation of 4-hydroxybenzophenone in different solvents. Photochem Photobiol Sci 14:2087-2096. https://doi.org/10.1039/c5pp00214a

24. Bhasikuttan C, Singh K, Palit DK, Sapre V, Mittal JP (1998) Laser flash photolysis studies on the monohydroxy derivatives of benzophenone. J Phys Chem A 5639:3470-3480 doi: S1089-5639(97)02375-X CCC

25. Das PK, Encinas MV, Scaiano JC (1981) Laser flash photolysis study of the reactions of carbonyl triplets with phenols and photochemistry of p-hydroxypropiophenone. J Am Chem Soc 103:4154-4162. https://doi.org/10.1021/ja00404a029

26. Palit DK (2005) Photophysics and excited state relaxation dynamics of $p$-hydroxy and $p$-amino-substituted benzophenones: a review. Res Chem Intermediates 31:205-225. https:// doi.org/10.1163/1568567053147020

27. Barsotti F, Ghigo G, Berto S, Vione D (2017) The nature of the light absorption and emission transitions of 4-hydroxybenzophenone in different solvents. A combined computational and experimental study. Photochem Photobiol Sci. https://doi. org/10.1039/C6PP00272B

28. Yoshino J, Kano N, Kawashima T (2013) Fluorescent azobenzenes and aromatic aldimines featuring an $\mathrm{N}-\mathrm{B}$ interaction. Dalton Trans:15826-15834. https://doi.org/10.1039/c3dt51689j

29. Saylam A, Seferoğlu Z, Ertan N (2014) Azo-8-hydroxyquinoline dyes: the synthesis, characterizations and determination of tautomeric properties of some new phenyl- and heteroarylazo-8-hydroxyquinolines. J Mol Liq 195:267-276. https://doi. org/10.1016/j.molliq.2014.02.027

30. Satam MA, Raut RK, Sekar N (2013) Fluorescent azo disperse dyes from 3-(1,3-benzothiazol-2-yl)naphthalen-2-ol and comparison with 2-naphthol analogs. Dyes Pigments 96:92-103. https://doi.org/10.1016/j.dyepig.2012.07.019

31. Puchtler H, Sweat F, Gropp S (1967) An investigation into the relation between structure and fluorescence of azo dyes. J Royal Microsc Soc 87:309-328. https://doi. org/10.1111/j.1365-2818.1967.tb04513.x

32. Wakatsuki Y, Yamazaki H, Grutsch PA, Santhanam M, Kutal C (1985) Study of intramolecular sensitization and other excitedstate pathways in orthometallated azobenzene complexes of Palladium (II). J Am Chem Soc 4:8153-8159. https://doi. org/10.1021/ja00312a057

33. Shimomura M, Kunitake T (1987) Fluorescence and photoisomerization of azobenzene-containing bilayer membranes. J Am Chem Soc 33:5175-5183. https://doi.org/10.1021/ja002 $51 \mathrm{a} 022$

34. Ghedini M, Pucci D, Crispini A, Aiello I, Barigelletti F, Gessi A (1999) Dinuclear cyclopalladated azobenzene complexes: a comparative study on model compounds for organometallic liquid-crystalline materials. Appl Organomet Chem 13:565-581. https://doi.org/10.1002/(SICI)1099-0739(199908)13:8<565::AIDAOC891>3.0.CO;2-C 
35. Han M, Hara M (2005) Intense Fluorescence from light-driven self-assembled aggregates of nonionic azobenzene derivative. J Am Chem Soc 127:10951-10955. https://doi.org/10.1021/ ja0509275

36. Aiello I, Ghedini M, La Deda M (2002) Synthesis and spectroscopic characterization of organometallic chromophores for photoluminescent materials: cyclopalladated complexes. JOL 96:249-259. https://doi.org/10.1016/S0022-2313(01)00229-0

37. Han MR, Hara M (2006) Chain length-dependent photoinduced formation of azobenzene aggregates. N J Chem:1-5. https://doi.org/10.1039/b511462d

38. Kano N, Yamamura M, Kawashima T (2015) 2,2'-Disilylazobenzenes featuring double intramolecular nitrogensilicon coordination: a photoisomerizable fluorophore. Dalton Trans 44:16256-16265. https://doi.org/10.1039/C5DT02038G

39. Warde U, Sekar N (2017) NLOphoric mono-azo dyes with negative solvatochromism and in-built ESIPT unit from ethyl 1,3-dihydroxy-2-naphthoate: estimation of excited state dipole moment and pH study. Dyes Pigments 137:384-394. https://doi.org/10.1016/j.dyepig.2016.10.032

40. Jadhav AG, Kothavale S, Sekar N (2017) Red emitting triphenylamine based rhodamine analogous with enhanced Stokes shift and viscosity sensitive emission. Dyes Pigments 138:5667. https://doi.org/10.1016/j.dyepig.2016.11.021

41. Mallion KB, Turner RW, Todd AH. United States Patent 0 "ice 3,714,16, 1967.

42. Du H, Kim C, Corradini MG, Ludescher RD, Rogers MA (2014) Micro-viscosity of fluid oil confined in colloidal fat crystal networks. Soft Matter 10:8652-8658. https://doi.org/10.1039/ C4SM01632G

43. Hazen T, Wuertz S (2004) Environmental biotechnology. Curr Opin Biotechnol 23:414

44. Malomo D, Abimbade SA, Oluwaseun AK (2017) Likely mechanism of dye adhesion on fabrics 1. Likely Mech Dye Adhes Fabrics:9-15

45. Ludescher RD, Jennifer S, Komaiko MGC (2012) Monoazo dyes as probes for bulk and local viscosity. Agro Food Ind Hi Tech 23:12-14

46. Cembran A, Bernardi F, Garavelli M, Gagliardi L, Orlandi G (2004) On the Mechanism of the cis-trans isomerization in the lowest electronic states of azobenzene: S0, S1, and T1. J Am Chem Soc 126:3234-3243. https://doi.org/10.1021/ja038 $327 y$

47. Rau H (1973) Spectroscopic properties of organic azo compounds. AngewChemInternatEdit 12:224-235

48. Laikhter A, City I, Behlke MA, Roberts W, City I. (19) United States (12) 2009;1.

49. Bloomfield A, Dewan RK (1971) Viscosity of liquid mixtures. J Phys Chem 75:3113-3119

50. Frisch MJ, Trucks GW, Schlegel HB, Scuseria GE, Robb MA, Cheeseman JR et al (2009) Gaussian 09, Revision C.01. Gaussian 09, Revision B01. Gaussian, Inc., Wallingford, CT

51. Becke AD (1993) Density-functional thermochemistry. III. The role of exact exchange. J Chem Phys 98:5648. https://doi. org/10.1063/1.464913

52. Becke AD (1988) Density-functional exchange-energy approximation with correct asymptotic behavior. Phys Rev A 38:30983100. https://doi.org/10.1103/PhysRevA.38.3098

53. Zhao Y, Truhlar DG (2008) The M06 suite of density functionals for main group thermochemistry, thermochemical kinetics, noncovalent interactions, excited states, and transition elements: two new functionals and systematic testing of four M06class functionals and 12 other function. Theor Chem Accounts 120:215-241. https://doi.org/10.1007/s00214-007-0310-x.

54. Otutu JO, Efurhievwe EM (2013) Synthesis of heterocyclic monoazo disperse dyes derived from 4-Amino-2-chloro-6, 7-dimethoxyquinazoline. J Appl Sci 13:924-928. https://doi. org/10.3923/jas.2013.924.928

55. Ezeribe Al, Bello KA, Adamu HM, Chindo I, Boryo YD (2013) Synthesis and dyeing properties of novel bifunctional reactive bromophenyl-1,3-thiazol-2-amine on nylon fabric. Am J Eng Res 3:52-61. https://doi.org/10.5901/ajis.2014.v3n5p125

56. Ziegler E, Zigeuner G (1949) Spaltungen mittels diazoniumverbindungen. Sci Pharm 17:113-117

57. Patil SR, Choudhary AS, Sekar N (2015) Disperse styryl and azo dyes for polyester and nylon fibre: synthesis, optical properties having the 1,2,4-triketo naphthoquinone skeleton. Fibers Polym 16:1068-1074. https://doi.org/10.1007/s12221-015-1068-8

58. MacNeil JD, Safe S, Hutzinger O (1976) The ultraviolet absorption spectra of some chlorinated biphenyls. Bull Environ Contam Toxicol 15:66-77. https://doi.org/10.1007/BF01686197

59. Brown WG, Reagan H (1947) Steric effects in the ultraviolet absorption spectra of aromatic nitro compounds. J Am Chem Soc 69:1032-1033. https://doi.org/10.1021/ja01197a016

60. Anpo M, Kamat PV (2010) Nanostructure science and technology. In: Masakazu Anpo PVK (ed) Enviromentally benign photocatalysts: applications of titanium oxide-baseed materials. Springer Science \& Business Media, p 382

61. Johnston LJ, Hugnot DJ, Wintgen VG (1988) Diphenylketyl radicals: spectroscopy, kinetics, and mechanisms. J Am Chem Soc 110:518-524

62. Tredwell CJ (1980) Viscosity dependent internal conversion in the rhodamine dye, fast acid violet 2R. J Chem Soc Faraday Trans II 76:1627-1637. https://doi.org/10.1039/f29807601638

63. Das K, Sarkar N, Ghosh AK, Majumdar D, Nath DN (1994) Excitedstate intramolecular proton transfer in 2-(2'-Hydroxyphenyl) benzimidazole and-benzoxazole: effect of rotamerism and hydrogen bonding. J Phys Chem 1:9126-9132. https://doi. org/10.1021/j100088a006

64. Biczok L, Berces T, Inoue H (1999) Effects of molecular structure and hydrogen bonding on the radiationless deactivation of singlet excited fluorenone derivatives. Chem Eur J 103:3837-3842. https://doi.org/10.1021/jp990208a

65. Martin MM (1975) Hydrogen bond effects on radiationless electronic transitions in xanthene dyes. Chem Phys Lett 35:105-111. https://doi.org/10.1016/0009-2614(75)85598-9

66. Mashaly HM, Abdelghaffar RA, Kamel MM, Youssef BM (2014) Dyeing of polyester fabric using nano disperse dyes and improving their light fastness using $\mathrm{ZnO}$ nano powder. Indian J Sci Technol 7:960-967

67. Lee M, Lee MS, Wakida T, Tokuyama T, Inoue G, Ishida S (2005) Chemical modification of nylon 6 and polyester fabrics by ozone-gas treatment. J Appl Polym Sci 100:1344-1348. https:// doi.org/10.1002/app.23382

68. Merian E, Carbonell J (1963) UL and VS. The saturation values, rates of dyeing, rates of diffusion, and migration of disperse dyes on heat-set polyester fibers. J Soc Dye Colour 79:505-514

69. Otutu JO (2013) Synthesis and properties of some hetaryl disazo and trisazo disperse dye derivatives of 2-Amino-5-nitrothiazole and 1-naphthylamine. Int J Recent Res Appl Studies 15:197-202

70. Sartori MF (2008) Spectral and fastness properties of benzothiazolylazo dyes. J Soc Dye Colour 83:144-146. https://doi. org/10.1111/j.1478-4408.1967.tb02756.x

71. Deshmukh MS, Sekar N (2014) A combined experimental and TD-DFT investigation of three disperse azo dyes having the nitroterephthalate skeleton. Dyes Pigments 103:25-33. https ://doi.org/10.1016/j.dyepig.2013.10.035

72. Ezeribe Al, Bello KA, Adamu HM, Chindo I, Boryo YD (2013) Synthesis and dyeing properties of novel bifunctional reactive bromophenyl-1,3-thiazol-2-amine on nylon fabric. Am J Eng Res 02:52-61 
73. Becerir B, Alpay HR. Ultraviolet (UV) protection of textiles: a review. International Scientific Conference 19-20 November 2010, Gabrovo, 2010, p. 301-11

74. Krzysztof K, Barbara LS (2014) Effect of selected metrological parameters of dyed polyester textiles on their UV-barrier properties. Fibers Polym 15:2077-2085. https://doi.org/10.1007/ s12221-014-2077-8

75. Hustvedt G, Crews P (2005) The ultraviolet protection factor of naturally-pigmented cotton. J Cotton Sci 9:47-55

76. Saylam A, Seferoglu Z, Ertan N (2008) Synthesis and spectroscopic properties of new hetarylazo 8-hydroxyquinolines from some heterocyclic amines. Dyes Pigments 76:470-476. https:// doi.org/10.1016/j.dyepig.2006.10.005
77. Shinde S, Sekar N (2019) Synthesis, spectroscopic characteristics, dyeing performance and TD-DFT study of quinolone based red emitting acid azo dyes. Dyes Pigments 168:12-27. https://doi. org/10.1016/j.dyepig.2019.04.028

78. Parr RG, Szentpaly LV, Liu S (1999) Electrophilicity index. J Am Chem Soc 121:1922-1924. https://doi.org/10.1021/ja983494x

Publisher's Note Springer Nature remains neutral with regard to jurisdictional claims in published maps and institutional affiliations. 